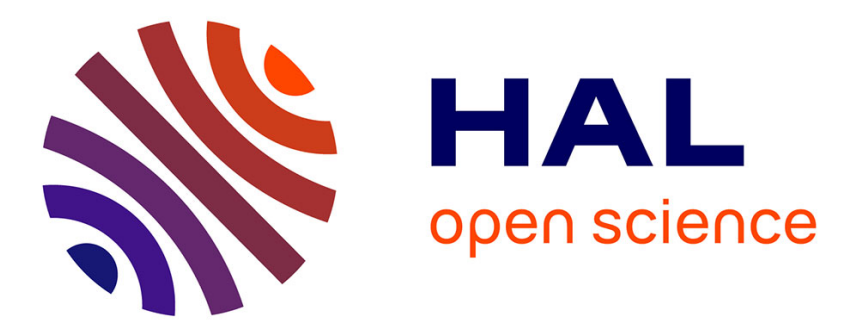

\title{
Lattice models applied to cyclic behavior description of quasi-brittle materials: Advantages of implicit integration.
}

Maxime Vassaux, Benjamin Richard, Frédéric Ragueneau, Alain Millard, Arnaud Delaplace

\section{To cite this version:}

Maxime Vassaux, Benjamin Richard, Frédéric Ragueneau, Alain Millard, Arnaud Delaplace. Lattice models applied to cyclic behavior description of quasi-brittle materials: Advantages of implicit integration.. International Journal for Numerical and Analytical Methods in Geomechanics, 2014, pp.43. 10.1002/nag.2343 . hal-01177051

\section{HAL Id: hal-01177051 \\ https://hal.inria.fr/hal-01177051}

Submitted on 20 Jul 2015

HAL is a multi-disciplinary open access archive for the deposit and dissemination of scientific research documents, whether they are published or not. The documents may come from teaching and research institutions in France or abroad, or from public or private research centers.
L'archive ouverte pluridisciplinaire HAL, est destinée au dépôt et à la diffusion de documents scientifiques de niveau recherche, publiés ou non, émanant des établissements d'enseignement et de recherche français ou étrangers, des laboratoires publics ou privés. 


\section{Lattice models applied to cyclic behavior description of quasi-brittle materials: advantages of implicit integration}

ARTICLE in INTERNATIONAL JOURNAL FOR NUMERICAL AND ANALYTICAL METHODS IN GEOMECHANICS · DECEMBER 2014

Impact Factor: $1.56 \cdot$ DOI: 10.1002/nag.2343

CITATION

1
DOWNLOADS

31
VIEWS

39

5 AUTHORS, INCLUDING:

Maxime Vassaux

Ecole normale supérieure de Cachan

6 PUBLICATIONS 2 CITATIONS

SEE PROFILE

F. Ragueneau

Ecole normale supérieure de Cachan

68 PUBLICATIONS 383 CITATIONS

SEE PROFILE

\section{Benjamin Richard}

Atomic Energy and Alternative Energies Com...

39 PUBLICATIONS 88 CITATIONS

SEE PROFILE 


\title{
Lattice models applied to cyclic behavior description of quasi-brittle materials: Advantages of implicit integration
}

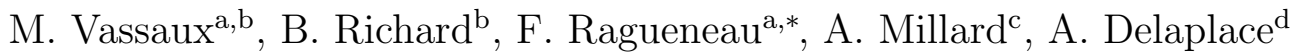 \\ ${ }^{a}$ LMT-Cachan, ENS-Cachan/CNRS/UPMC/PRES Universud Paris, 61 avenue du \\ President Wilson, 94230 Cachan, France \\ ${ }^{b}$ CEA, DEN, DANS, DM2S, SEMT, Laboratoire d'Études de Mécanique Sismique, \\ F-91191 Gif-sur-Yvette, France \\ ${ }^{c} C E A, D E N$, DANS, DM2S, SEMT, Laboratoire de Mecanique Systèmes et Simulation, \\ F-91191 Gif-sur-Yvette, France \\ ${ }^{d}$ Lafarge Centre de Recherche, 95 rue du Montmurier, 38291 Saint Quentin Fallavier, \\ France
}

\begin{abstract}
Comprehension and quantification of quasi-brittle materials behavior requires complex experiments when focusing on cyclic or multi-axial loadings. As an alternative, virtual testing, which can be computed using lattice discrete elements models (LDEM), is particularly interesting. LDEM already provide a physical description of the quasi-brittle materials behavior, but further attention has to be paid to numerical integration. LDEM are explicitly integrated, such integration has been proven in the literature to be accurate when cracking is involved, by means of efficient schemes such as the "Saw-tooth" algorithm. In order to extend the range of application of LDEM to more complex loading paths, such as compressive or cyclic loadings, involving contact and friction mechanisms, qualitativeness as well as quantitativeness of explicit integration has to be assessed anew. We hereby propose an implicit quasi-static integration scheme for LDEM based on specific non-linearities encountered in quasi-brittle materials, namely contact and fracture, to circumvent expected stability and accuracy issues. Efficiency of both schemes is investigated by means of simulations of a uniaxial cyclic test and a com-
\end{abstract}

\footnotetext{
${ }^{*}$ Corresponding author

Email address: ragueneau@lmt.ens-cachan.fr (F. Ragueneau)
} 
pression test.

Keywords: lattice model, discrete elements, fracture, contact, quasi-brittle material, implicit integration

\section{Introduction}

In the last two decades, important advances have been achieved in the field of quasi-brittle materials behavior modeling. These materials can be considered as cohesive granular assemblies and then modeled with the discrete element method (DEM), originally introduced by Cundall and Strack [1]. Transition from former work for non-cohesive media [1], to cohesive ones has been inspired by the lattice models initially developed by Kawai [2]. Lattice models were designed to limit computation costs by discretizing matter as a simple network of small rigid-bodies interacting through springs. Direct applications of lattice models were realized on medias subject to cracking, such as concrete [4, 5], or even more ductile materials, such as steel [3]. Fracture and related mechanisms, such as size-effect, in cohesive granular assemblies have been also intensively investigated $[7,6]$.

The use of the DEM, and therefore accounting for contact is a necessary addition to lattice models when aiming at investigating multi-axial or cyclic loading paths.

In recent LDEM contact is considered. Depending on the particle shape, the contact force is respectively modeled as a function of the distance between particles centroids [9], or as the overlapping area of the contacting particles $[10,11]$, whether the particles are circular or polygonal.

The choice of the particles shape is important. While in finite element methods (FEM) the mesh is just a formal discretization, in DEM the mesh and subsequently the particles shape has a physical meaning. Polygonal particles are more suitable to represent mechanisms involving cracks, such as contact and friction. A more representative crack roughness is generated, comparing to spherical particles, which is important in order to study crack closure mechanisms. However, even in presence of polygonal particles, the fact of not considering constitutive laws can only be achieved for excessive particles size refinement, leading to unacceptable computational costs. Nevertheless refinement is sufficient to reproduce cracks coarse roughness and tortuosity. Consequently, the addition of the simple Coulomb friction model - to ac- 
count for mircoscale roughness - enables to capture phenomena observed at the macroscopic scale. Additionally, spurious geometrical porosity is avoided [12], and a more realistic representation of macroscopic phenomena such as breaking through bending and dilatancy can be achieved [13]. On that account, for cohesive granular assemblies, such as concrete, polygons are often chosen, in spite of an increased computational complexity in terms of contact detection, of force determination, and, at last what will be the purpose of this paper, of equilibrium integration.

LDEM description of quasi-brittle materials behavior is simple and physically meaningful, however it implies the use of unregularized and non-smooth constitutive laws. In the present work, these constitutive laws refer to fracture and contact mechanisms. Non-smooth constitutive laws force us to pay attention to the quality of the results given by the integration scheme. Indeed, in the case of explicit integration schemes, the use of excessively small time-steps is prerequisite to ensure accuracy and stability.

The classic dynamic framework used in DEM is not a satisfactory option. The proposed LDEM is therefore developed in the quasi-static framework. Indeed, the use of the dynamic framework requires the identification of arbitrary parameters such as the damping ratio. In addition, the use of the quasi-static framework allows studying crack propagation phenomena while avoiding dependencies related to wave propagation effects, such as reflection, highly influenced by material heterogeneities. Material heterogeneities which are outside the scope of this work, and not described by the model.

Classic applications of lattice models concern crack opening modes (either mode I or mixed modes I and II) and monotonic loadings. In these applications contact is not predominant, thus explicit integration schemes provide accurate results, and therefore are commonly used. Among explicit integration schemes, the "Saw-tooth" algorithm developed in [20] is very popular. The "Saw-tooth" method provides stability of the results, even in the case of brittle behavior. In the case of more complex loading paths and particularly cyclic loadings, crack closure occurs. Contact becomes a predominant force transfer mechanism alongside cohesion. In extreme situations when long cracks close, numerous contacts can be initiated simultaneously.

Therefore, in the present framework of quasi-static simulations, inaccu- 
racies due to explicit integration are amplified by the absence of dynamic viscous damping leading to oscillations. With the aim of extending lattice models to cyclic or multi-axial loading paths, the efficiency and limits of explicit integration in the quasi-static framework have to be assessed.

It is well accepted with other methods such as Contact Dynamics (CD) [14] or Discontinuous Deformation Analysis (DDA) [15], that implicit integration is required to obtain qualitative as well as quantitative results when contact is involved. The use of numerical damping or excessively small timesteps to obtain converged values of either contact or frictional forces is not mandatory[16]. However, integration algorithms used for these methods developed in a dynamic framework cannot be retained. In consequence, the purpose of the present paper is to investigate in which measure the explicit "Saw-tooth" integration, which has proven to be efficient with respect to fracture description, remains applicable within the LDEM framework while conserving a high-level accuracy when contact is considered.

To circumvent stability issues brought in by the unregularized contact constitutive laws, we propose to develop a robust fully implicit quasi-static integration scheme specifically designed for LDEM applied to quasi-brittle materials subjected to cyclic loadings. The implicit integration scheme is implemented in accordance with the definition of the two important nonlinearities present in the model, namely fracture and contact. The integration scheme is a combination of the same sequentially linear algorithm "Saw-tooth" algorithm [20], for the treatment of fracture; and of an iterative predictor-corrector method, to compute forces equilibrium.

The paper is outlined as follows. First, the LDEM, on which this study is based, is described. Details are given on particles definition, forces computation and the failure criterion. The implicit procedure based on an original predictor is then introduced. Finally efficiency investigations of the "Sawtooth" explicit and of the proposed implicit integration are led by means of simulations of a cyclic tension test and a compression test, implying local multi-axial solicitations. 


\section{The lattice discrete elements model}

In the present section, a brief description of the mechanisms considered in the model is established. Mechanisms include cohesion, contact, frictional sliding and fracture. Gravity is not considered due to the quasi-static framework, even as a static force. Regarding cohesion and fracture, description is based on the 2D version of the discrete elements code DEAP [17].

\subsection{Particles geometry and meshing}

As mentioned in the introduction, polygonal shaped particles allow a more realistic description of quasi-brittle materials. In spite of an increased computation complexity, since the outline of particles cannot be analytically written, polygonal particles are still chosen. Discrete particles are obtained by decomposition of a volume using a Voronoi tessellation, implying that every particle is a convex polygon. Particles centroids are randomly distributed on a grid overlaying the sample [18], which size controls particles density (fig. 1a). This leads to irregularly shaped particles with relatively similar areas (fig. 1b). According to the following definition of interactions, and particularly cohesion, irregularly shaped particles and random centroids disposition will ensure general isotropy of the model's behavior.

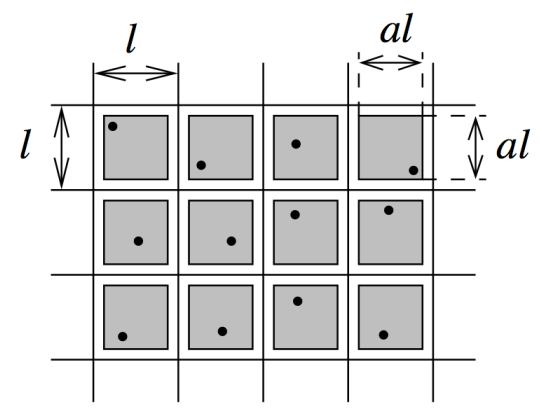

(a) Random disposition of centroid nodes in an actual area of size $(a l)^{2}$ [17]

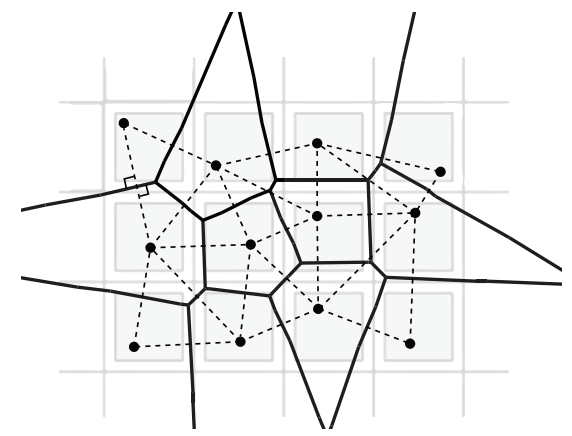

(b) Bi-dimensional polygonal mesh generated by Voronoi tessellation from the left centroids distribution

Figure 1: Mesh generation 


\subsection{Cohesion}

Cohesion interactions are introduced between particles by means of EulerBernoulli beams connecting particles centroids and which support normal and shear forces as well as bending moment. Consequently cohesion forces are point loads applied to the particles centroids. The cohesion is then defined as elastic depending linearly on centroids displacements $\underline{u}$ and rotation $\theta$. The cohesion forces and moment between two connected particles $i$ and $j$ are expressed as:

$$
\underline{F}_{c o h, i j}=\left\{\begin{array}{l}
F_{N, i j}=\frac{E A_{b, i j}}{l_{b, i j}}\left(\underline{u}_{i}-\underline{u}_{j}\right) \cdot \underline{n}_{b, i j} \\
F_{T, i j}=\frac{12 E I_{b, i j}}{l_{b, i j}^{3}}\left(\underline{u}_{i}-\underline{u}_{j}\right) \cdot \underline{t}_{b, i j}-\frac{6 E I_{b, i j}}{l_{b, i j}^{2}}\left(\theta_{i}-\theta_{j}\right) \\
M_{Z, i j}=\frac{6 E I_{b, i j}}{l_{b, i j}^{2}}\left(\underline{u}_{j}-\underline{u}_{i}\right) \cdot \underline{t}_{b, i j}+\frac{4 E I_{b, i j}}{l_{b, i j}}\left(\theta_{i}-\frac{\theta_{j}}{2}\right)
\end{array}\right.
$$

where $\underline{n}_{b, i j}$ and $\underline{t}_{b, i j}$ are normal and tangential vector of the cross-section $A_{b, i j}$ of the beam, $E$ is the beam Young modulus, identical for every beam, $I_{b, i j}$ its moment of inertia, and $l_{b, i j}$ its initial length (fig. 2a).

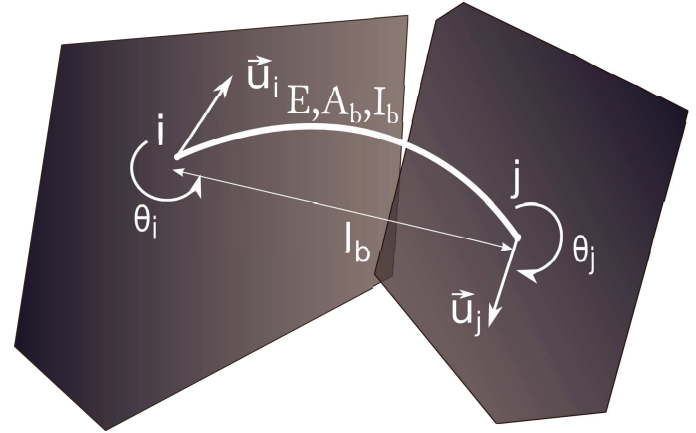

(a) Cohesion description

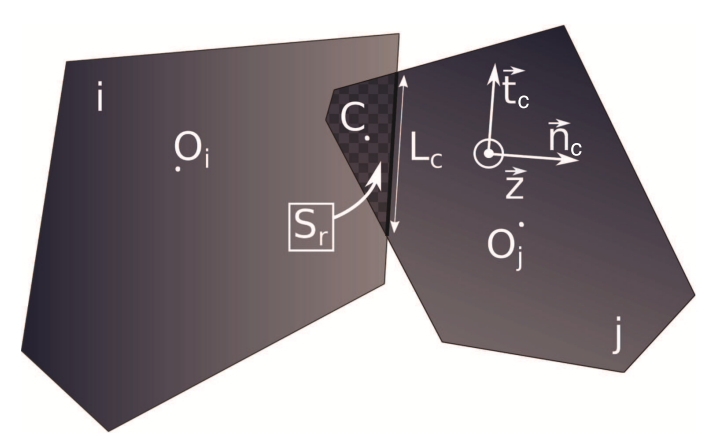

(b) Contact description

Figure 2: Interactions description

\subsection{Cracking}

In comparison with macroscopic models, fracture is straightforwardly described. When extension strains and rotations in the beam exceed the fol- 
lowing criterion (2), the beam is considered as broken and then, is removed from the system. The failure of beams is perfectly brittle.

$$
\begin{gathered}
P_{i j}=\left(\frac{\epsilon_{i j}}{\epsilon_{c r}}\right)+\left(\frac{\left|\theta_{i}-\theta_{j}\right|}{\theta_{c r}}\right)<1 \\
\text { with } \quad \epsilon_{i j}=\frac{u_{i}-u_{j}}{l_{b, i j}}
\end{gathered}
$$

where $\epsilon_{c r}$ and $\theta_{c r}$ are material parameters which define the perfectly brittle behavior of the beams of the lattice model. For every beam, the couple of parameters is statistically determined by means of a Weibull distribution [7], identified by inverse fitting tension and compression simulations with experimental force-displacement curves [21]. The Weibull distribution is defined as:

$$
f(x, \lambda, k)=\frac{k}{\lambda}\left(\frac{x}{\lambda}\right)^{k-1} \mathrm{e}^{-(x / \lambda)^{k}}
$$

where $f$ is the value to be computed statistically (i.e $\epsilon$ or $\theta$ ); $x$ is a random number (different for every link); $\lambda$ and $k$ are two parameters called respectively scale and shape factor, which define the Weibull distribution.

\subsection{Contact}

Contact interactions are unilateral, they are only introduced when two particles overlap and are not linked with a cohesive beam. In the DEM, particles are perfectly rigid bodies, consequently contact force between two particles is computed as a function of the overlapping area. Other properties of the contact resultant force, such as contact forces directions or application point, are also deduced from the intersection of the two overlapping particles.

\subsubsection{Detection}

Before computing contact forces it is necessary to detect overlapping. Since particles are polygonal, intersection of particles can not be solved analytically as with discs, numerical detection is required. Overlap detection is a time-consuming process, increasing quickly with regard to the number of particles. In order to limit detection costs, intersection search is restricted to close neighbors [22].

The complete description of the polygonal intersection is computed through an algorithm developed to detect overlap of convex polygons [23]. The following contact properties are computed from the intersection polygon (fig. $2 \mathrm{~b})$ : 
- Intersection area $S_{r}$ as the area of the intersection polygon.

- Contact band width $L_{c}$ as the longest segment inside contained in the intersection polygon.

- Contact normal $\overrightarrow{n_{c}}$ and tangential $\overrightarrow{t_{c}}$ directions as the normal and tangential directions to the longest segment in the intersection polygon.

- Application point $C$ of contact forces as the center of inertia of the intersection polygon.

\subsubsection{Constitutive contact model}

Now that the contact geometrical features are known, the contact force model can be introduced. The contact force model is also similar to beam theory. It is a simple description of the contact force, which does not account for the bi-dimensional aspect of particles, unlike Hertz's theory for contacts between circular particles. Complex models do exists, such as energy-based models [24], but beam models are still mostly used. Due to their simplicity and representativity, they reveal to be very convenient for elastic contact force computation between polygonal particles [10, 11].

A slight modification is made in the classical normal force formula in beam theory to introduce the overlap area $S_{r}$.

$$
\begin{aligned}
\underline{F}_{c o n t, i j} & =\frac{E A_{i j}}{l_{c, i j}}\left(\Delta \underline{u}_{i j} \cdot \underline{n}_{c, i j}\right) \underline{n}_{c, i j} \\
& =\frac{E\left(L_{c, i j} \times 1\right)}{l_{c, i j}} \times\left(-\frac{S_{r, i j}}{L_{c, i j}}\right) \underline{n}_{c, i j} \\
& =-\frac{E S_{r, i j}}{l_{c, i j}} \underline{n}_{c, i j}
\end{aligned}
$$

where $l_{c}$ is a characteristic length supposed to be the initial length of the beam in the beam theory, but here it has no exact signification.

The length $l_{c}$ is directly obtained from the particles dimensions. In the idea of considering the bi-dimensional shape of particles, $l_{c}$ is often computed as a function of diameters of the circles of equivalent area of the contacting particles. The formula is the inverse of the sum of the inverses of the equivalent diameters $D_{i}, D_{j}$ [11]. Here particles have similar dimensions, so we want $l_{c}$ to be an average of both diameters (8).

$$
\frac{1}{l_{c}}=\frac{1}{2}\left(\frac{1}{D_{i}}+\frac{1}{D_{j}}\right)
$$




$$
\text { Thus if } \quad D_{i}=D_{j}=D \quad \text { then } \quad l_{c}=D
$$

\subsection{Frictional sliding}

A Coulomb's type of friction is considered introducing the friction coefficient $\mu$ :

$$
\underline{F}_{f r i c, i j}=\min \left(\frac{E I_{c, i j}}{\left(l_{c, i j}\right)^{3}}\left[\left(\underline{u}_{c, i j}-\underline{u}_{c, j i}\right) \cdot \underline{t}_{c, i j}-\Delta u_{s, i j}\right], \mu\left\|\underline{F}_{c o n t, i j}\right\|\right) \underline{t}_{c, i j}
$$

The elastic part, before reaching the perfectly plastic behavior of the friction force, is computed as the tangential force in beam's theory, except that the displacement used are the displacements accumulated since contact has begun, $\underline{u}_{c}$, instead of total displacements $\underline{u}$ :

$$
\underline{u}_{c, i j}^{t+1}= \begin{cases}\underline{u}_{c, i j}^{t}+\underline{\delta}_{i}^{t} & \text { if } i \text { and } j \text { are overlapping } \\ 0 & \text { if } i \text { and } j \text { are distant }\end{cases}
$$

$\underline{u}_{c, i j}$ is the accumulated displacement of the particle $i$ since the contact with particle $j$ has begun. The moment of inertia $I_{c}$ is computed with a beam's thickness equals to $L_{c}: I_{c}=\frac{1 \times L_{c}^{3}}{12}$. A new internal variable $\Delta u_{s}$ measuring the sliding relative displacement between two particles is introduced and updated in order to verify Coulomb's criterion. Both internal variables $\underline{u}_{c}$ and $\Delta u_{s}$ are reset when contact is lost.

\subsection{Contact and friction induced moment}

Unlike cohesive beams, no flexural stiffness is explicitly considered as for cohesion interactions. Moment is induced by the change of application point of the contact forces from the center of mass $C$ to the centroids of respective particles $O_{i}$ and $O_{j}$.

$$
\underline{M}_{c f, i j}=\underline{O}_{i} C \wedge\left(\underline{F}_{c o n t, i j}+\underline{F}_{f r i c, i j}\right)
$$

\section{Implicit quasi-static integration scheme}

The LDEM presented here describes internal forces $\underline{F}_{\text {int }}$ non-linearly with respect to centroids displacements. Non-linearity of internal forces is due to the non-linear elastic contact forces, and the inelastic fracture and frictional 
sliding mechanisms.

In order to overcome stability issues of explicit integration schemes due to the consideration of contact and friction mechanisms, a fully implicit integration scheme is proposed. Integration is computed iteratively such that equilibrium of internal forces and external forces $\underline{F}_{\text {ext }}$ at time-step $t+1$ can be written:

$$
\underline{F}_{i n t}^{t+1}\left(\underline{u}^{t+1}\right)=\underline{F}_{e x t}^{t+1}
$$

Due to the iterative process, implicit integration implies that attention must be paid to robustness. Consequently, the implicit integration scheme is split in two parts: on one hand the computation of forces equilibrium considering cohesion, contact and friction interactions; and on the other one the evolution of the fracture mechanism.

Equilibrium is computed using an implicit predictor-corrector method, while the evolution of fracture is computed using the efficient "Saw-tooth" algorithm [20].

Handling aside the fracture mechanism helps to simplify the predictive part of equilibrium's integration, which does not have to account for cracking's evolution anymore.

In addition to the separation of the computation of forces equilibrium and fracture evolution, numerical relaxation is introduced to overcome remaining robustness issues.

While the development of the model in a quasi-static framework allows lower computation costs, giving up inertia and viscosity effects introduces the problem of rigid body motion. Disconnected particles, from the main structure on which no displacements boundary conditions are defined, require a specific treatment that is developed after the description of the whole integration scheme.

Note that in the present section, normalization of results is done with respect to maximum values reached during the simulations. Besides, the time rather refers to pseudo-time than to real time, since quasi-static computations are considered. 


\subsection{Sequentially linear integration [20] of fracture mechanism}

The fracture mechanism in LDEM consists in progressively removing cohesive links and updating the global stiffness matrix, while loading conditions evolve. At every load increment, the elastic solution is computed with the stiffness matrix updated after the previous load increment, and cohesive links exceeding their elastic limit is removed.

The "Saw-tooth" algorithm developed by Rots [20] is here applied to LDEM [17]. This "Saw-tooth" algorithm efficiency lies in its specific way of removing cohesive links. The main difference with classic integration schemes, is that equilibrium is computed after each beam removal. Equilibrium is then solved as a succession of linear problems with respect to the cracking problem.

In the present model, the fracture mechanism is also combined with other non-linear mechanisms, thus the load is not driven by the rupture of cohesive links, unlike the original "Saw-tooth" algorithm. A classic incremental time-stepping is still used.

The link to be removed at the current iteration, is defined as the link that exceeds the most its elastic limit. Here is a description of an iteration of the failure integration algorithm:

1. Beginning of iteration $m+1$ at time-step $t$

2. Iterative resolution of equilibrium and actualization of the global displacement: $\underline{u}^{t, m+1}=\underline{u}^{t, m}+\underline{\delta u}^{t, m+1}$

3. Computation of the inverse of the failure criterion of each link between two particles $i$ and $j: \alpha_{i j}=\frac{1}{P_{i j}}$

4. If $\min _{i, j} \alpha_{i j}>1$ move to time-step $t+1$ and $\underline{\underline{K}}_{c o h}^{t+1,0}=\underline{\underline{K}}_{c o h}^{t, m}$, otherwise continue to the next step.

5. The link with the lowest $\alpha$ is considered broken and the global cohesive stiffness matrix is updated: $\underline{\underline{K}}_{c o h}^{t, m+1}=\underline{\underline{K}}_{c o h}^{t, m}-{ }^{t} \underline{\underline{T}}_{i j} \underline{\underline{K}}_{c o h, i j}^{l o c} \underline{\underline{T}}_{i j}$ where the link between $i$ and $j$ has the lowest $\alpha$ value.

6. Proceed to the next iteration at the time-step $t$ and go back to step 1 .

Note that the implementation of such algorithm is greatly helped by the linear elastic and perfectly brittle behavior of cohesive links. The "Saw-tooth" 
algorithm becomes heavy in terms of computational costs in presence of more complex constitutive laws [25], because the number of non-linear "events" grows rapidly. Nevertheless, attempts have been pursued for plastic behaviors $[26]$.

\subsection{Implicit equilibrium's integration}

As aforementioned forces equilibrium is computed considering cohesion, contact and frictional interactions. Equilibrium equations are solved iteratively with a predictor-corrector method. Emphasis is here put on the choice of an appropriate predictor depending on the type of non-linearities.

Since cracking state remains constant during equilibrium computation, cohesion is linear elastic. Friction is modeled as a perfectly plastic behavior defined by Coulomb's law. Consequently only contact reveals to be troublesome.

\subsubsection{Contact non-linearities}

Contact non-linearities have two distinct origins. The first is due to the geometric description of the media, namely the polygonal shape of particles, and thus of their intersection. The second origin is mechanical, due to the unilateral aspect of contact.

The contact normal force is defined as a function of the overlap of two contacting particles. However, the degrees of freedom of the system are the displacements of particles centroids. The polygonal and randomly defined shape of particles makes it impossible to compute analytically as a function of the centroids original position, displacements and rotation. In addition, since particles are irregularly shaped, the relation between the intersection and the normal relative displacement is neither smooth nor even monotonic. In comparison, for discs, although it is also non-linear, the intersection's evolution is smooth, strictly monotonic, and is only a function of normal relative displacement of centroids.

As an illustration, a comparison of the evolution of the intersection area with the relative normal displacement of centroids, between polygonal and circular shaped particles is exposed in figure 3. The motion is at first simple attraction, and latter (after the dashed line) a combination of rotation and attraction. The introduction of rotation induces temporarily a diminution of the intersection area for the polygonal particles, while for the circular ones 
it has no influence.

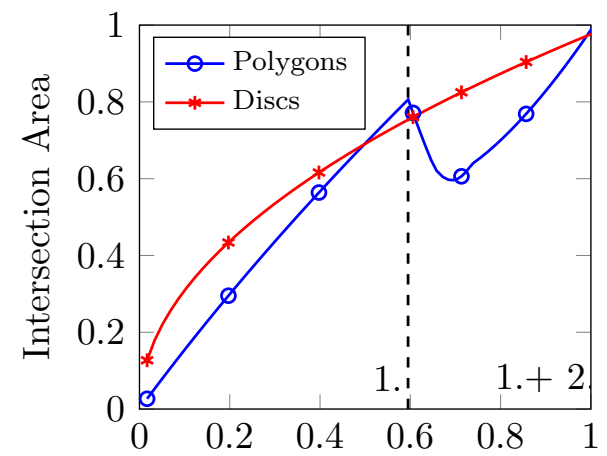

Normal relative displacement

(a) Evolution of the normalized intersection area with the normal relative displacement

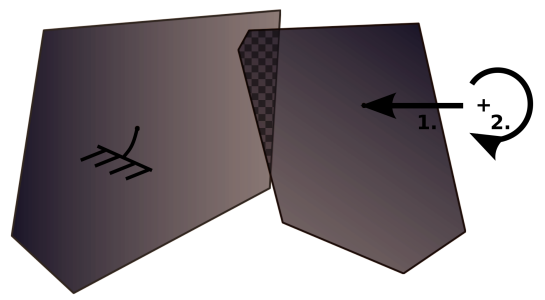

(b) Particles motion

Figure 3: Geometrical non-linearity due to particles shape

The mechanical non-linearity due to the unilateral aspect of contact causes continuity issues around the contact initiation point. According to the contact model based on beam theory, the contact stiffness can be written: $k_{c}=E L_{c} / l_{c}$, where $L_{c}$ is the contact intersection width. For vertex-to-edge contacts (see fig. 4a), the first derivative of the contact force (i.e. the contact stiffness) with respect to indentation $\left(S_{r} / L_{c}\right)$ is continuous, but the second is not. However for edge-to-edge contacts (see fig. 4b), there is no more continuity of the first derivative, since $L_{c}$ jumps from zero to a finite value. This particular continuity issue will be highlighted during latter simulations (see $\S 3.2 .4)$.

Non-monotonic evolutions and discontinuities can lead to numerical singularities or instabilities. Indeed, the positive definition of the stiffness matrix might not be ensured. Or when contact is initiated or lost, oscillations or convergence issues can occur, since equilibrium can not be reached properly.

\subsubsection{Predictor's definition: Linearized contact model}

Classically in the DEM the centroids displacements are predicted without having to inverse the stiffness matrix, displacements are predicted from 


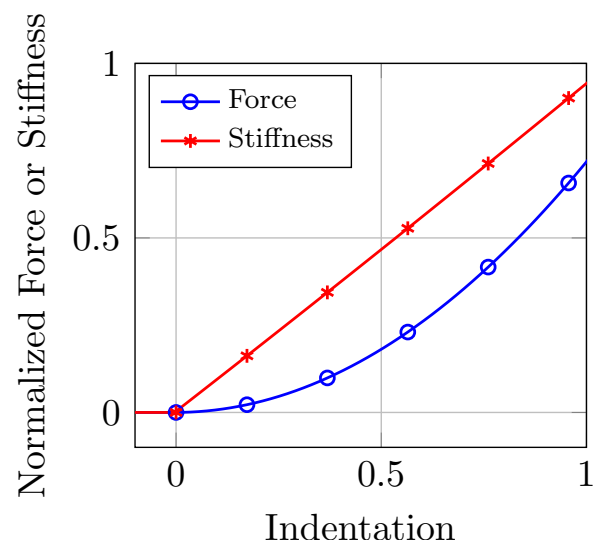

(a) Vertex-to-edge contact

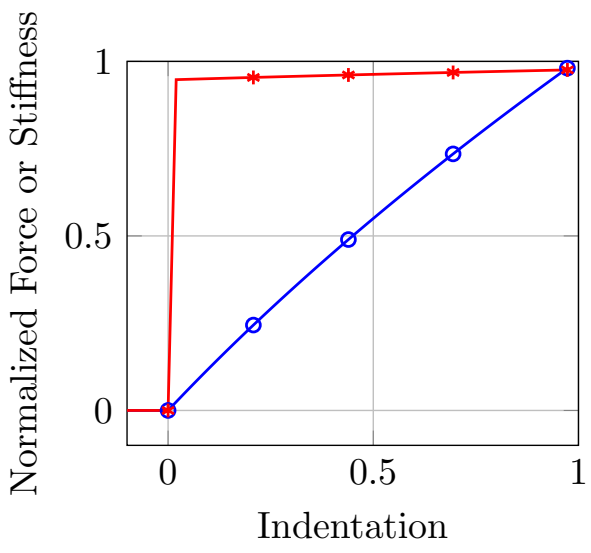

(b) Edge-to-edge contact

Figure 4: Mechanical non-linearity due to the unilateral aspect of contact: Normalized normal force and stiffness evolutions with the normalized indentation

accelerations of the previous time-step. In the quasi-static framework such information is not available. Then the predictor step consists in determining the centroids displacements with simplifying assumptions on the definition of interactions. Internal forces are defined as a linear function of centroids displacements:

$$
\underline{\tilde{F}}_{i n t} \simeq \underline{\underline{\tilde{K}}} \cdot \underline{u}
$$

Since in LDEM cohesion is predominant on the rest of interactions, for sake of simplicity a prediction only accounting for cohesion forces, which are linear by definition, has been attempted first:

$$
\underline{\underline{\tilde{K}}}=\underset{i=1, \ldots, n_{\text {particles }}}{\boldsymbol{A}}\left(\sum_{j=1, \ldots, n_{\text {beam }}(i)}\left(\underline{t}^{t} \underline{\underline{T}}^{i j} \underline{\underline{K}}_{c o h}^{l o c, i j} \underline{\underline{T}}^{i j}\right)\right)
$$

where $\underline{\underline{\underline{K}}}$ is the global predictive stiffness matrix, $j$ indices referring to the particles cohesively linked to the particle $i . \underline{\underline{T}}_{i j}$ is the transformation matrix from the local referential of the contact $\left(\underline{n}_{b, i j}, \underline{t}_{b, i j}, \underline{z}\right)$ to the global referential $(\underline{x}, \underline{y}, \underline{z})$, with $\alpha$ the angle $\left(\underline{n}_{b, i j}, \underline{x}\right)$ : 


$$
\underline{\underline{T}}_{i j}=\left[\begin{array}{cccccc}
\cos (\alpha) & \sin (\alpha) & 0 & 0 & 0 & 0 \\
-\sin (\alpha) & \cos (\alpha) & 0 & 0 & 0 & 0 \\
0 & 0 & 1 & 0 & 0 & 0 \\
0 & 0 & 0 & \cos (\alpha) & \sin (\alpha) & 0 \\
0 & 0 & 0 & -\sin (\alpha) & \cos (\alpha) & 0 \\
0 & 0 & 0 & 0 & 0 & 1
\end{array}\right]
$$

And the 6x6 local stiffness $\underline{\underline{K}}_{c o h}^{l o c, i j}$ is computed directly from the linear cohesion model:

$$
\underline{\underline{K}}_{c o h}^{l o c, i j}=\left[\begin{array}{cccccc}
\frac{E A_{b}}{l_{b}} & 0 & 0 & -\frac{E A_{b}}{l_{b}} & 0 & 0 \\
& \frac{12 E I_{b}}{l_{b}^{3}} & -\frac{6 E I_{b}}{l_{b}^{2}} & 0 & -\frac{12 E I_{b}}{l_{b}} & \frac{6 E I_{b}}{l_{b}^{2}} \\
& & \frac{4 E I_{b}}{l_{b}} & 0 & \frac{6 E I_{b}}{l_{b}^{2}} & -\frac{2 E I_{b}}{l_{b}} \\
& & & \frac{E A_{b}}{l_{b}} & 0 & 0 \\
& & & & \frac{12 E I_{b}}{l_{b}^{3}} & 0 \\
& & & & & \frac{4 E I_{b}}{l_{b}}
\end{array}\right]
$$

As shown on a simple test (fig. 5) oscillations occur. Contact is successively detected and lost after every iteration of prediction-correction and convergence can not be found. A prediction of contact and friction contributions is necessary.

On the basis of the "open-close" iteration introduced by Shi [8], a linearized contribution of contact and friction $\underline{\underline{K}}_{c o n t}^{l o c}$ is introduced in the global predictive stiffness matrix, if contact has been detected at the previous iteration. This implies that the global predictive stiffness matrix is updated after each iteration.

$$
\begin{aligned}
& \underline{\underline{\tilde{K}}}=\underset{i=1, \ldots, n_{\text {particles }}}{\boldsymbol{A}}\left(\sum_{j=1, \ldots, n_{\text {beam }}(i)}\left({ }^{t} \underline{\underline{T}}^{i j} \underline{\underline{K}}_{c o h}^{l o c, i j} \underline{\underline{T}}^{i j}\right)+\right. \\
& \left.\sum_{l=1, \ldots, n_{\text {contact }}(i)}\left({ }^{t} \underline{\underline{T}}^{i l} \underline{\underline{\tilde{K}}}_{c o n t}^{l o c, i l} \underline{\underline{T}}^{i l}\right)\right)
\end{aligned}
$$


where $l$ indices refer to the particles overlapping the particle $i$ at the previous iteration.

In comparison with finite element methods, Lagrange multipliers or their derivatives [27], could have been used to account linearly for contact between two particles. However, in discrete elements an overlap has to be allowed to depict macroscopic deformation since particles are perfectly rigid, thus an alternative prediction based on penalization methods has been chosen.

Since the contact model is already very similar to a beam model, the prediction of contact forces is done introducing a beam of thickness equals to the width of the overlap area $L_{c}$ and of length $l_{c}$. In the end it is equivalent to the following assumptions:

- For the contact force: the indentation increment is assumed equal to the normal relative displacement increment of the two particles in contact:

$$
\delta \tilde{F}_{c o n t, i j}=\frac{E L_{c, i j}}{l_{c, i j}}\left(\delta \underline{u}_{i}-\delta \underline{u}_{j}\right) \cdot \underline{n}_{i j}
$$

- For the friction force: the elastic prediction is simply identical to the exact expression, since the increment of cumulated displacements is the same as the increment of total displacements:

$$
\delta \underline{u}_{c, i j}=\delta \underline{u}_{i} \quad \Rightarrow \quad \delta \tilde{F}_{f r i c, i j}=\frac{E I_{c, i j}}{\left(l_{c, i j}\right)^{3}}\left(\delta \underline{u}_{i}-\delta \underline{u}_{j}\right) \cdot \underline{t}_{i j}
$$

- For the torque induced by contact and friction forces: actually no parallel can be made with beam theory since moment is computed as the transposition of the contact resultant force from the inertia center of the contact overlap area to the respective centroids. Nevertheless the introduction of a beam flexural stiffness is still chosen and allows relative improvements of the predictions when bending moment is considered.

$$
\delta \tilde{M}_{c f, i j}=\frac{4 E I_{c}}{l_{c}}\left(\delta \theta_{i}-\frac{\delta \theta_{j}}{2}\right)
$$

To recap, here is the $6 \times 6$ local predictive stiffness matrix used for contact 
and friction interactions:

$$
\underline{\underline{K}}_{c o n t}^{l o c, i j}=\left[\begin{array}{cccccc}
\frac{E L_{c}}{l_{c}} & 0 & 0 & -\frac{E L_{c}}{l_{c}} & 0 & 0 \\
& \frac{12 E I_{c}}{l_{c}^{3}} & 0 & 0 & -\frac{12 E I_{c}}{l_{c}} & 0 \\
& & \frac{4 E I_{c}}{l_{c}} & 0 & 0 & -\frac{2 E I_{c}}{l_{c}} \\
& & & \frac{E L_{c}}{l_{c}} & 0 & 0 \\
& \text { sym. } & & & \frac{12 E I_{c}}{l_{c}^{3}} & 0 \\
& & & & & \frac{4 E I_{c}}{l_{c}}
\end{array}\right]
$$

With such prediction, the predictor-corrector method implemented here is close to Newton's solution methods. The prediction could be qualified as secant since it accounts for the evolution of contact properties.

A tangent prediction has been avoided because of the strong non-linearities. Indeed the evolution of the overlap area with respect of nodal displacements is not monotonic we can not be sure of the positive definition of the tangent stiffness matrix.

On the same test, which showed the necessity of a prediction accounting for contact and friction (fig. 5), convergence is now found easily.

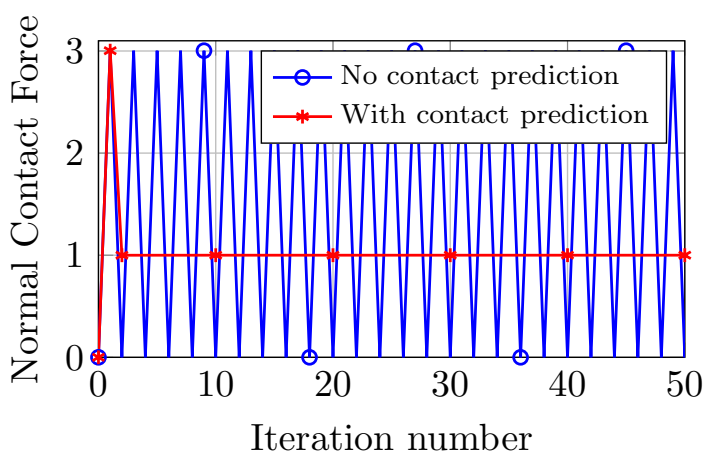

(a) Convergence of the contact force

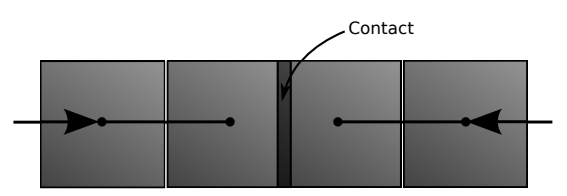

(b) Simulation setup

Figure 5: Influence of prediction on convergence: Evolution of normalized normal contact force (with the applied force) with iteration count

\subsubsection{Numerical relaxation}

Due to the non-linearity classified as mechanical, the model is subject to convergence issues when contact is initiated or lost, because of discontinuities of first or second order in the contact behavior. Damping is usually a 
practical way to overcome oscillation issues, but since dynamic effects have been disregarded, we have chosen to introduce numerical relaxation.

Relaxation is done, only considering a certain amount of the predicted displacement increment, if the residue increases or decreases too slowly:

$$
\underline{u}^{k+1}=\underline{u}^{k}+h^{k+1} \delta \underline{u}^{k+1} \quad h^{k+1}=\left\{\begin{array}{l}
h^{k} \text { if } \delta \underline{r}^{k+1} \leq 10^{-4} \\
\frac{h^{k}}{2} \text { if } \delta \underline{r}^{k+1}>10^{-4}
\end{array}\right.
$$

where $\delta u^{k+1}$ is the predicted increment, $\delta r^{k+1}$ is the residue increment and $h^{k+1}$ is the relaxation factor at the current iteration $k+1$.

\subsubsection{Local validation of the equilibrium integration procedure}

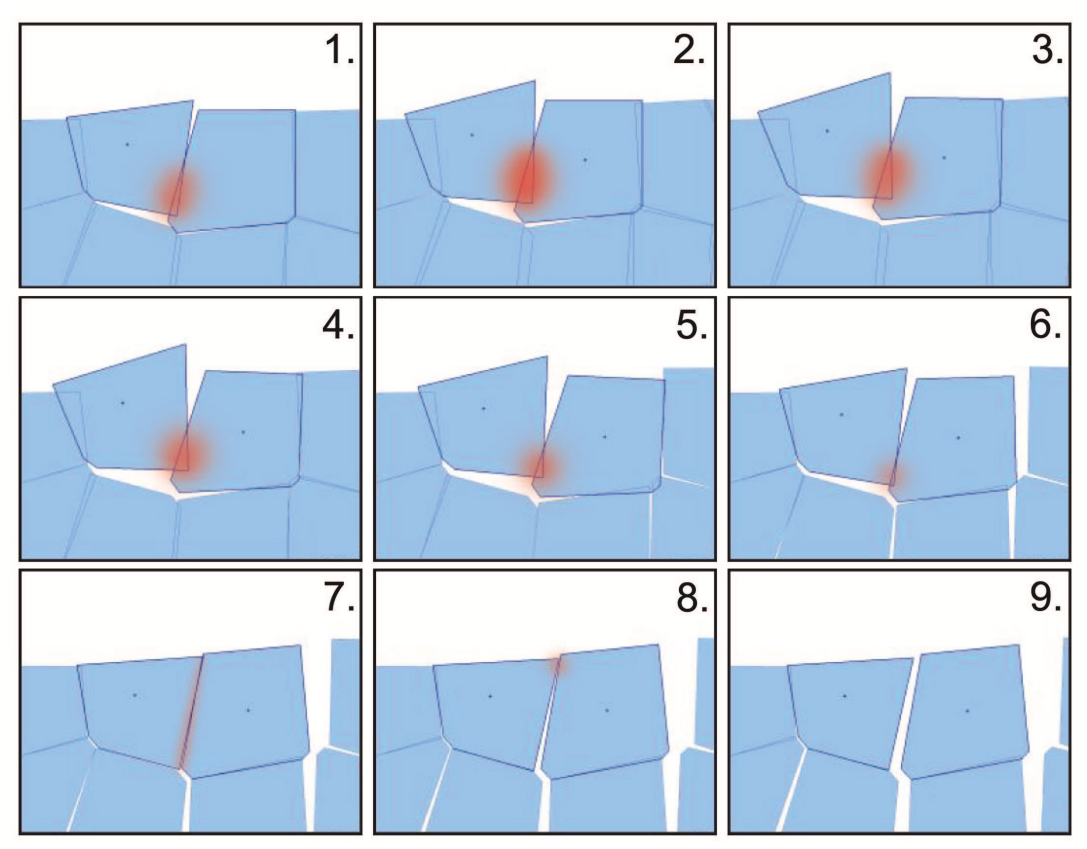

Figure 6: Motion of the two contacting particles

In order to show the efficiency of the chosen predictor as well as the introduction of relaxation, we study the motion of contacting particles during a simple local test. A 5x5 particles precracked square is subject to successive compressive and tensile loadings. The sample is randomly precracked in different locations so as to observe contacts, without having to activate 
the failure mechanisms during the simulation, and therefore introducing additional bias. We focus exclusively on the contact behavior of two particles. The figure 6 shows the motion of the two contacting particles (particles with bold orange edges at the center of the pictures), contact is represented by the dark spot.

During the test important non-linearities are observed. Geometrical nonlinearity of contact behavior is activated due to rotations of the two particles induced by structural effects. The mechanical non-linearity is also activated when contact is almost lost and is only maintained on a thin layer: from the time-step 1200 (frame 6) to the time-step 1700 (frame 8). In this time lapse, as we can see from the motion of the particles, the contact overlap location between the two particles passes from the bottom to the top of the particles, before being completely lost.

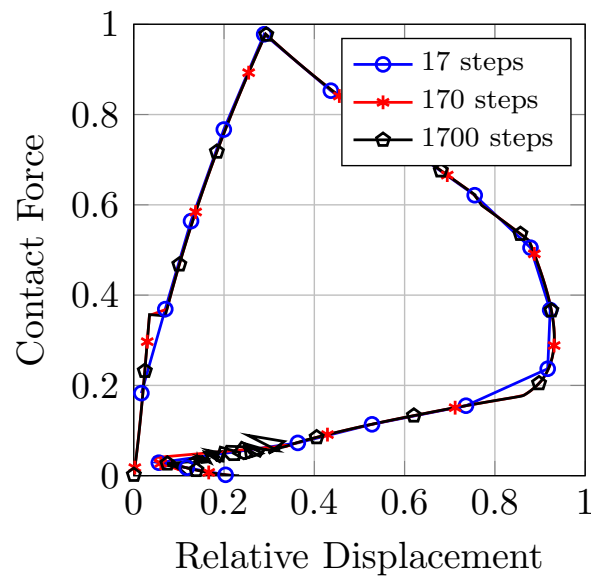

(a) Without relaxation

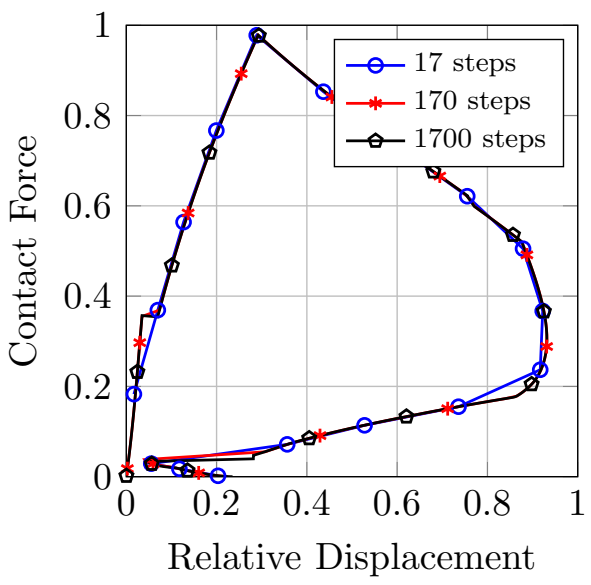

(b) With relaxation

Figure 7: Time-stability of the proposed implicit integration: Evolution of normalized normal contact force with the normalized normal relative displacement

The simulation shows stability with respect to time-step size of the implicit algorithm without numerical relaxation (fig. 7). Whatever the discretization the response follows the same-path. However at the end of the simulation, some convergence issues of the predictor-corrector method are observed for the fine discretization due to the discontinuity when contact is lost. 


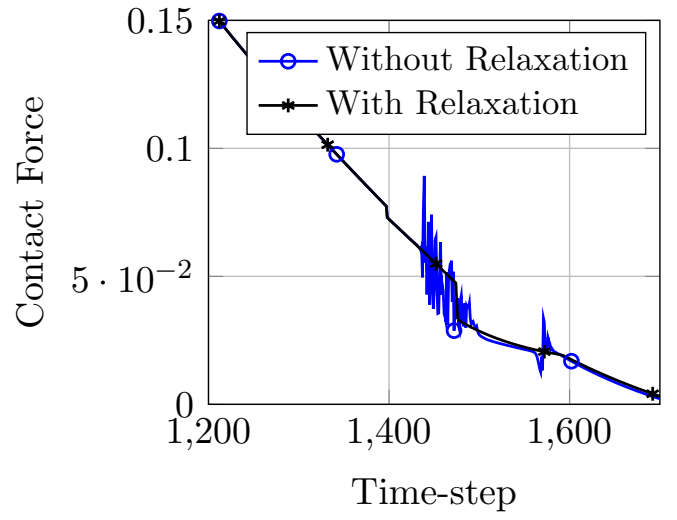

(a) Contact force evolution

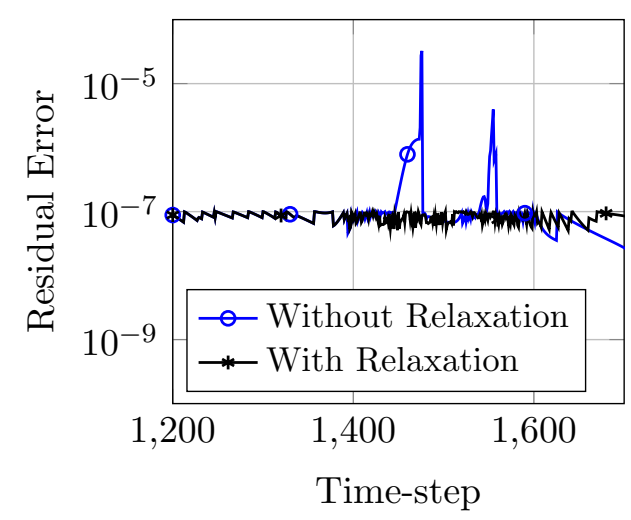

(b) Residual error evolution

Figure 8: Influence of relaxation on convergence when contact is lost: Evolution of normalized normal contact force and residual error with time

The figure 8 shows more in details what happens during the last 500 time-steps of the finely discretized simulation (1700 time-steps) in terms of contact force and residual error, around the point of loss of contact.

As observed during the time-stability study, the implicit integration without relaxation is subject to oscillations. In the case of the implicit integration we can see from the residue computation (fig. 8b) that during some time intervals convergence cannot be found (convergence being considered as reached for a residue value below $10^{-7}$ ). Oscillations might be explained by the multiple neighboring equilibrium solutions and the inability of integration schemes to stabilize in one. The addition of numerical relaxation to the implicit integration helps greatly to overcome this limitation, hence increasing the robustness of the implicit integration.

\subsection{Disconnected particles}

Spalling occurs when single particles or group of particles are completely disconnected from the main structure, in other words when a group of particles has no more cohesive links with the rest of the particles of the structure, on which boundary conditions are defined. If a group of particles is not directly or indirectly, through connectivities, subject to sufficient boundary conditions, rigid-body motions can result.

In the general case of DEM, disconnected particles are not a particular issue. The equilibrium is then usually solved in the dynamics framework, in 
which inertia and damping effects are considered, thus a unique solution of the problem exists even in presence of rigid-body motions. In the quasi-static framework rigid-body motion have to be prevented to preserve uniqueness of solution.

It has to be noticed that contact, unlike cohesion, cannot be considered as a link between two groups of particles due to its unilateral aspect. Indeed if contact is only applied on one point of a group of particles, and no cohesive link remains, the contact force generated is unbalanced. Therefore the group of particles is ejected and send away.

\subsubsection{Detection}

Detection of disconnected groups of particles is intricate. When a group of more than two particles is disconnected from the main structure, a detection criterion like if a particle has no more cohesive links, then it can be considered as disconnected, which would work for a group of one particle, does not hold anymore.

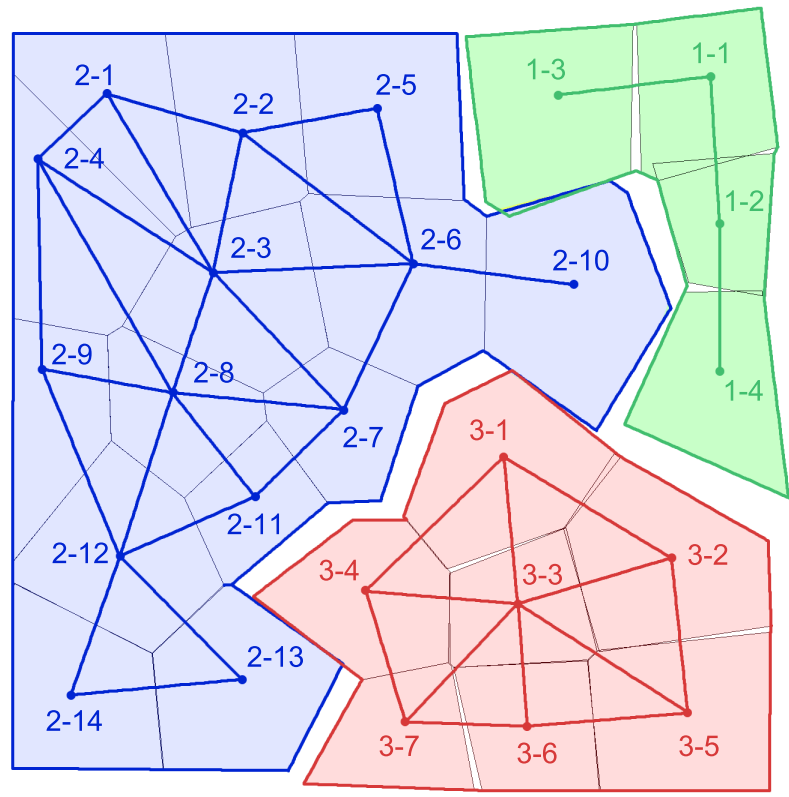

Figure 9: Breadth-First Search algorithm applied to LDEM: illustration of the exploration process. The coordinates $\mathrm{X}-\mathrm{Y}$ represent the order of discovery of the body $(\mathrm{X})$ and of the particle inside the body $(\mathrm{Y})$. 
Some help can be found in graph's theory which framework is similar to lattice models, namely nodes and connectivities. Graph's theory algorithms to detect connected components are practical to solve the issue of detecting disconnected groups of particles.

A connected component is a set of points in a graph, between which a direct or indirect path exists. In the case of LDEM, the points are the particles and the path a continuous succession of cohesive beams. Therefore a disconnected group of particles can be defined as a connected component in the graph that represents the particles of the whole structure.

Among available algorithms, to explore the whole set of particles and their connectivities, a simple and efficient algorithm has been chosen: the Breadth-First Search (BFS) algorithm [28]. The BFS algorithm research method consists in starting from one particle, explore each of its connected particle, and successively for each connected particle explore its unexplored connected particles. This is done recursively until no new particle is explored. A disconnected group of particles is then defined. This process is repeated starting from an unexplored particle in the mesh, until all the disconnected groups of particles are defined and each particle in the mesh has been explored. The figure 9 sums up the BFS algorithm process and shows a possible order of exploration of particles.

In the worst case, the BFS algorithm will explore each particle as many times as its number of connectivities. Therefore the time-complexity of the algorithm is depending on the density of cohesive links. In the most dense situation, in other words if all the particles are directly linked to each other, the time-complexity is $O\left(n^{2}\right)$, where $n$ is the number of particles in the structure.

Now that independent groups of particles are detected, possibility of rigidbody motion has to be determined. If an independent group of particles does not fulfill the following conditions, the specific treatment described in the following paragraph will have to be applied:

1. At least 3 of degrees of freedom with an applied boundary condition.

2. Boundary conditions applied on two different directions $\left(u_{x}\right.$ and $\left.u_{y}\right)$.

\subsubsection{Numerical treatment}

Unlike detection, treatment of disconnected particles is rather simple. The concerned particles are considered as non-interacting with the rest of 
the structure and then removed. This treatment expresses the fact that in most cases, spalling induces a direct loss of mass as disconnected pieces of quasi-brittle materials fall away from the structure. However this treatment might have some limitations in case of spalling occurring inside an horizontal crack, since disconnected particles might still have a mechanical influence. Another treatment could be to lock the disconnected group of particles until a contact between one of them and the rest of the structure is detected. But in some cases, for example if only a single contact force is applied to the group of disconnected particles, it can result in infinite and uncontrolled displacements, in absence of inertia or viscous effects to counterbalance the contact force. So the choice of removing disconnected particles has been kept.

\subsection{Global algorithm}

The solution algorithms for equilibrium and failure have been separately introduced. They are assembled in the global algorithm as follows:

$\rightarrow$ Loop on time steps $t$.

$\hookrightarrow$ Loop on the failure algorithm's iterations $m$ and disconnected particles checking.

$\hookrightarrow$ Loop on contact and friction algorithm's iterations $k$.

A detailed flowchart of the global algorithm is presented in the figure 10. Note that convergence of the predictor-algorithm is chosen to be reached for residual error below $10^{-7}$. This value showed good efficiency in terms of accuracy and time-costs. 


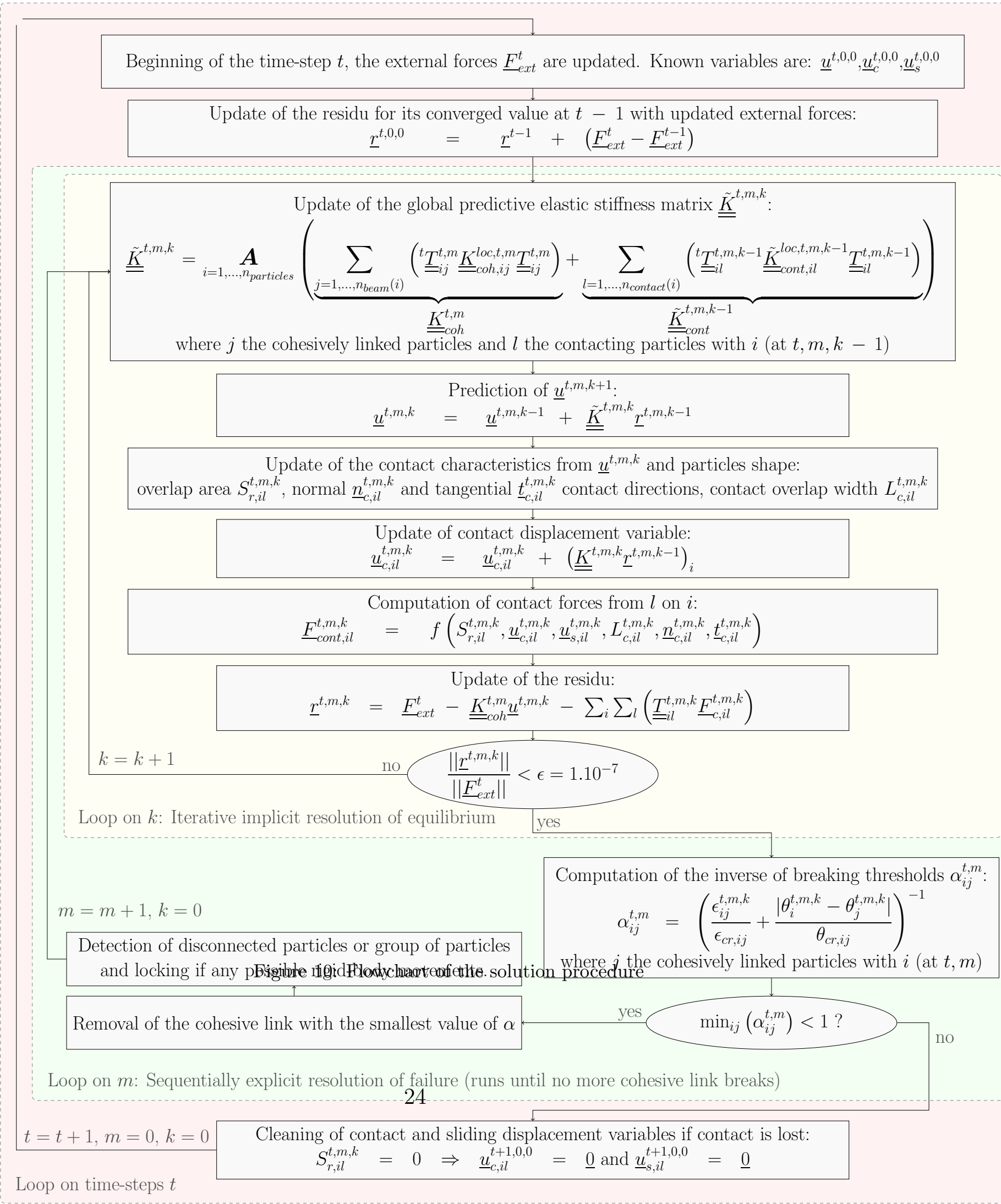




\section{Investigations on integrations efficiency of the concrete response under uniaxial cyclic tension and compression}

The purpose of the present LDEM is to be used to simulate multi-axial and cyclic loading paths, extending its range of application outside of monotonic loading paths and crack opening modes. It starts with being able to simulate uniaxial cyclic tension test and compression test.

Uniaxial cyclic tension and compression tests are interesting indicators of the efficiency of explicit "Saw-tooth" integration and of the developed implicit integration procedures. Indeed, in both tests contact and friction mechanisms are largely involved, however not in identical manners. Regarding uniaxial cyclic tension tests (fig. 11a) contact and friction mechanisms are involved separately from fracture mechanism. Cracking occurs during loading under tension while contact and friction occur during unloading.

Concerning compression tests (fig. 11b), at some point cracking and contact occur simultaneously. It coincides with the second and third stages of the failure mechanisms under compression as described by Rossi [29], when the columns of concrete parallel to the applied load formed by coalescing diffuse cracking collapse successively. At the local scale these mechanisms induce complex and multi-axial interactions between particles. Among available compression tests we have chosen the test with dry platen boundary conditions from Vonk [31].

\subsection{LDEM identification}

Simulations are computed on a square of $50 \times 50$ particles, which side measures $0.1 \mathrm{~m}$. At such particles density, convergence of the macroscopic elastic properties with respect to the particles density is already reached. Model's parameters value regarding the cohesive behavior as well as fracture mechanism are processed by inverse identification [21] on tension and compression experimental stress-strain behaviors. These two test provide rich enough information on the fracture behavior, involving mode I as well as mode II cracking modes.

Beam's Young modulus $E$ is chosen so as to obtain a macroscopic Young modulus of $40 \mathrm{GPa}$. Weibull distributions scale parameter $\lambda$ of breaking thresholds $\epsilon_{c r}$ and $\theta_{c r}$ are calibrated so as to match, respectively, tension and 


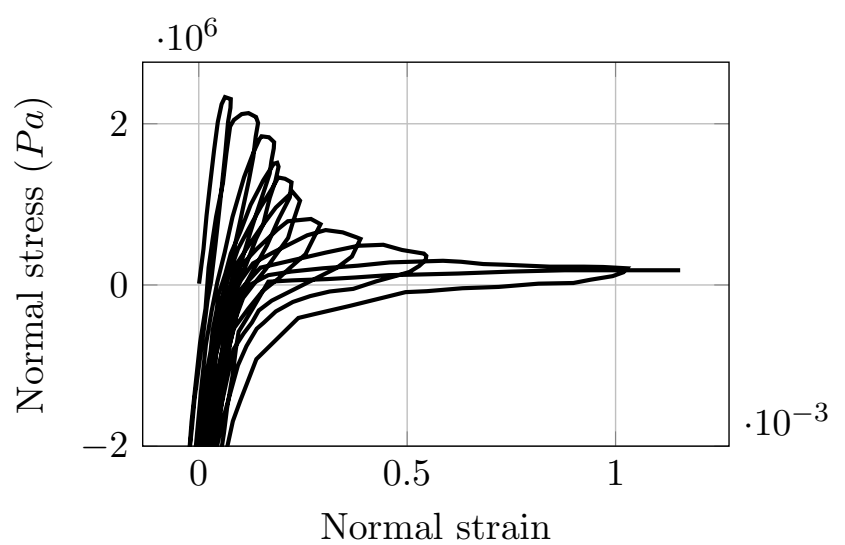

(a) Uniaxial cyclic tension test [30]

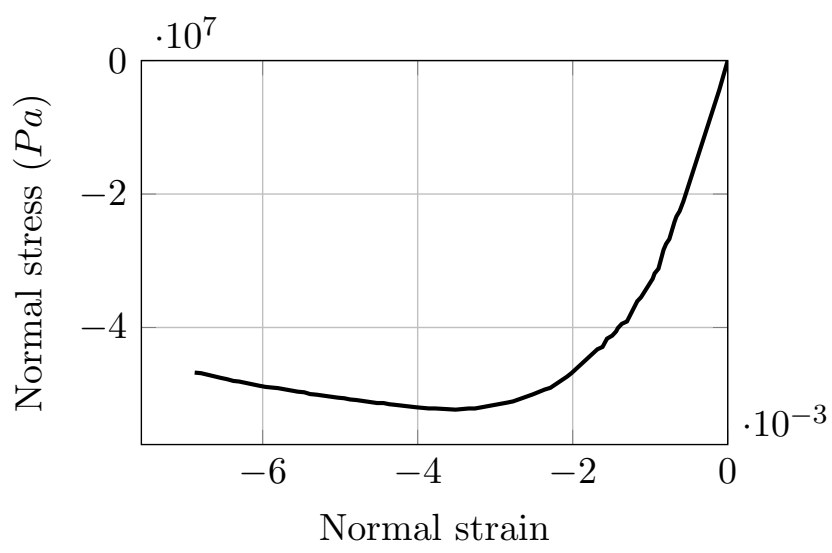

(b) Compression test [31]

Figure 11: Experimental stress-strain response of concrete

compression peak stresses and the unique $k$ for both distributions, is calibrated to fit tension post-peak softening behavior. Regarding contact and friction, contact's Young modulus is chosen identical to the cohesion one. Friction coefficient $\mu$ has been chosen following the consensus when it comes to concrete, since no distinction on the composition (cement or aggregate) of particles is made.

Parameters values are summed up in the table 1.

\begin{tabular}{c|c|c|c|c|c} 
& $E(\mathrm{~Pa})$ & $\lambda_{\epsilon_{c r}}$ & $\lambda_{\theta_{c r}}$ & $k$ & $\mu$ \\
\hline Parameter's value & $48 \times 10^{9}$ & $2.5 \times 10^{-4}$ & $9.17 \times 10^{-2}$ & 2.3 & 0.7
\end{tabular}

Table 1: Parameter values for the tests simulation

\subsection{Investigations methodology}

The loading versus pseudo-time for the uniaxial cyclic tension and compression tests is shown in fig. 12 . 


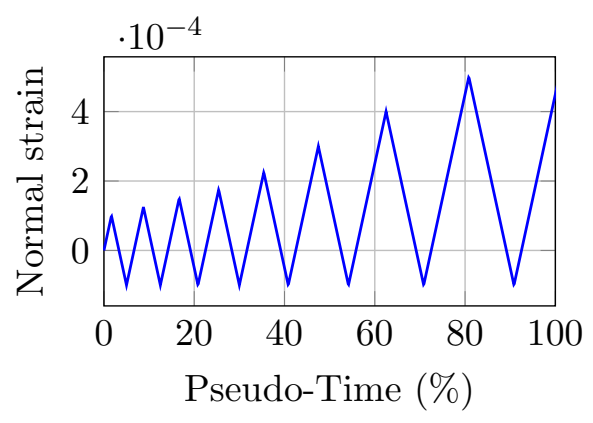

Pseudo-Time (\%)

$$
\text { uniaxial cyclic test }
$$

(a) Uniaxial cyclic tension test

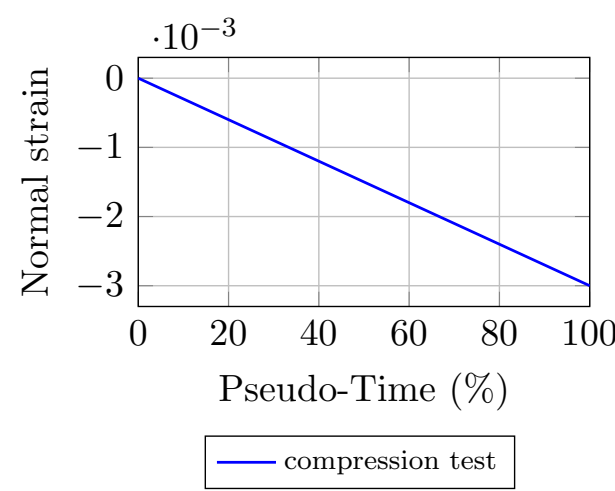

(b) Compression test

Figure 12: Evolution of loading with time

During the simulations, several outputs are analyzed:

- Evolution of normal stresses with respect to normal strains.

- Evolution of dissipated energy through cracking $E_{\text {frac }}^{t}$ with respect to pseudo-time, computed incrementally as follows [32]:

$$
E_{\text {frac }}^{t+1}=E_{\text {frac }}^{t}+\frac{1}{2}\left({ }^{T} \underline{u}^{t+1} \cdot \underline{\underline{K}}_{c o h}^{t} \cdot \underline{u}^{t}-{ }^{T} \underline{u}^{t} \cdot \underline{\underline{K}}_{c o h}^{t+1} \cdot \underline{u}^{t+1}\right)
$$

- Evolution of dissipated energy through friction $E_{\text {fric }}$ with respect to pseudo-time, computed incrementally as follows [32]:

$$
\begin{aligned}
& E_{\text {fric }}^{t+1}=E_{\text {fric }}^{t}+ \\
& \sum_{i=1, \ldots, n_{\text {particles }}}\left(\sum_{j=1, \ldots, n_{\text {contact }}(i)}\left(T\left(\delta \underline{F}_{f r i c, i j}\right)^{t+1} \cdot\left(\delta \Delta u_{s, i j} \underline{t}_{c, i j}\right)^{t+1}\right)\right)
\end{aligned}
$$

- Cracking patterns.

On one hand, the three first outputs provide global quantitative information on the computed response. The dissipated energies allow to investigate specifically the efficiency of integration schemes with regard to each nonlinear mechanism, distinguishing fracture from contact and friction. On the other hand, cracking patterns provide qualitative local information on the 
computed response and help to assess the physical significance of the results.

The simulations are carried out for different normal strain increment $\delta \epsilon$. Ranges of tested strain increments are defined so as to be able to follow variations of the applied load. In addition, excessively fine strain increments will not be tested because computation costs in terms of time and memory would be unreasonable. A summary of the computation costs is also provided (see fig.16a and 21a).

Simulations are realized with 10 different meshes and draws of the rupture thresholds, presented scalar results are averaged values over the 10 simulations.

\subsection{Uniaxial cyclic tension test: results and comments}

Simulations are carried out for strain increments of $\delta \epsilon=1.10^{-6}, \delta \epsilon=$ $1.10^{-7}$ and $\delta \epsilon=1.10^{-8}$. Regarding implicit integration, only simulations for strain increments of $\delta \epsilon=1.10^{-6}$ and $\delta \epsilon=1.10^{-7}$ are shown since no dependence to the strain increment size is observed.

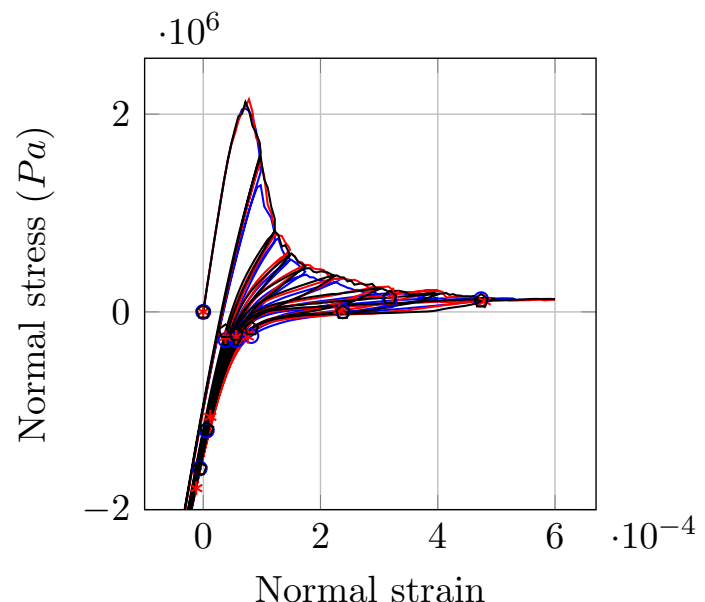

Normal strain

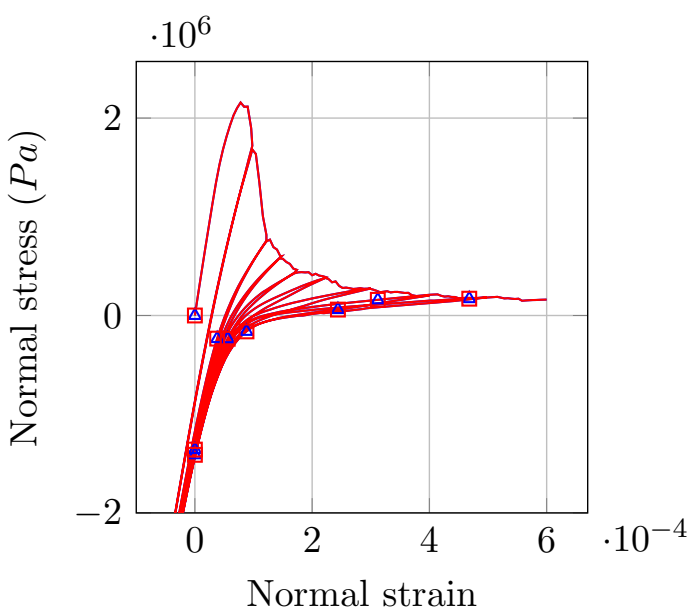

$\multimap$ exp. $\delta \epsilon=1.10^{-6} \multimap$ exp. $\delta \epsilon=1.10^{-7}$ $\checkmark$ exp. $\delta \epsilon=1.10^{-8}$

(a) Explicit integration

(b) Implicit integration

Figure 13: Evolution of the normal stress with respect to normal strain during the whole cyclic test 
The stress-strain curves (fig.13) obtained with explicit integration are similar independently of the strain increment, convergence is quickly obtained. Moreover these responses are close to the implicit response as well as to the experimental behavior (fig.11a).

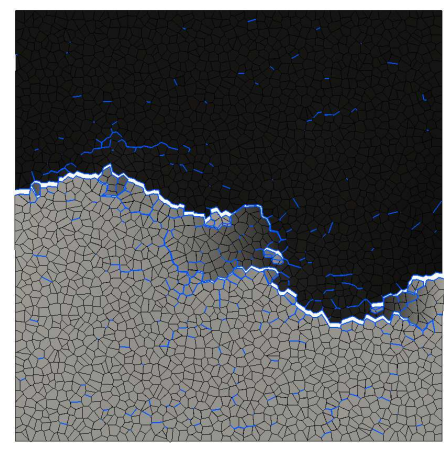

(a) Explicit: $\delta \epsilon=1.10^{-6}$

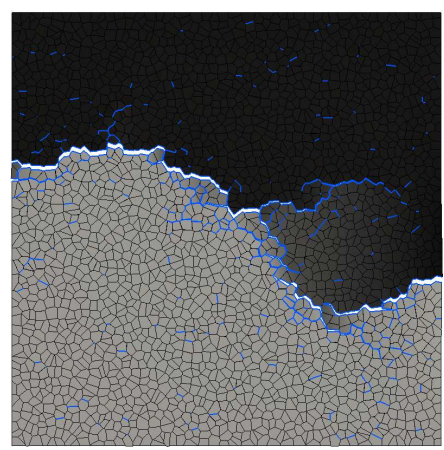

(d) Implicit: $\delta \epsilon=1.10^{-6}$

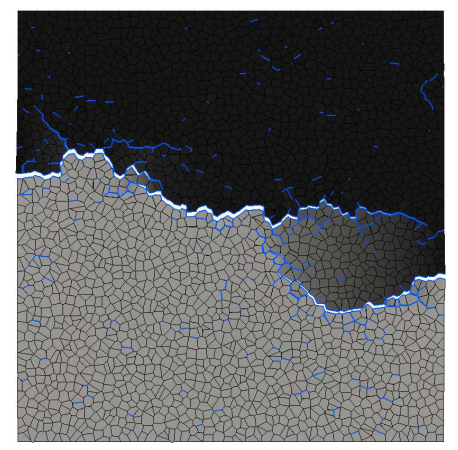

(b) Explicit: $\delta \epsilon=1.10^{-7}$

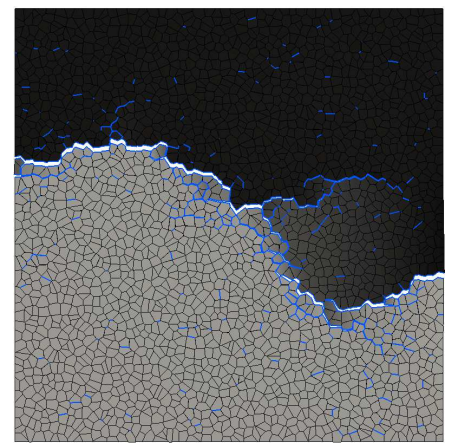

(e) Implicit: $\delta \epsilon=1.10^{-7}$

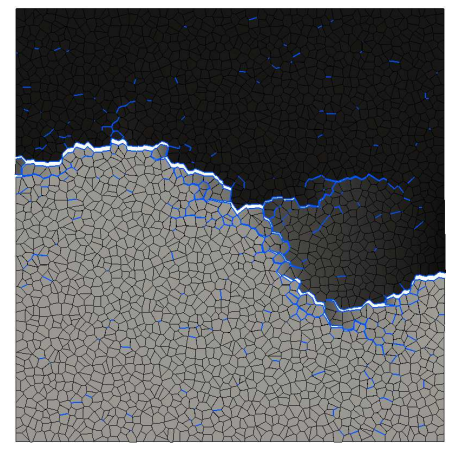

(c) Explicit: $\delta \epsilon=1.10^{-8}$

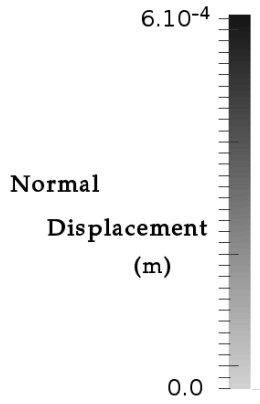

Figure 14: Cracking patterns after the cyclic test

The presented simulations of unixial cyclic test do not use notched samples, so as not to force localization. In result, field measurements such as cracking patterns are only influenced by the spatial variations of rupture thresholds which are identical for each strain increment. Similarly to the stress-strain responses, explicit cracking patterns (fig.14) converge toward implicit cracking patterns. In the case of monotonic tension tests cracking patterns are identical with explicit and implicit integration [20], independently of the strain increment. Here, in the case of cyclic tests, coupling 
with friction mechanisms influences the cracking process, and therefore implies strain increment refinement to converge towards identical cracking patterns.
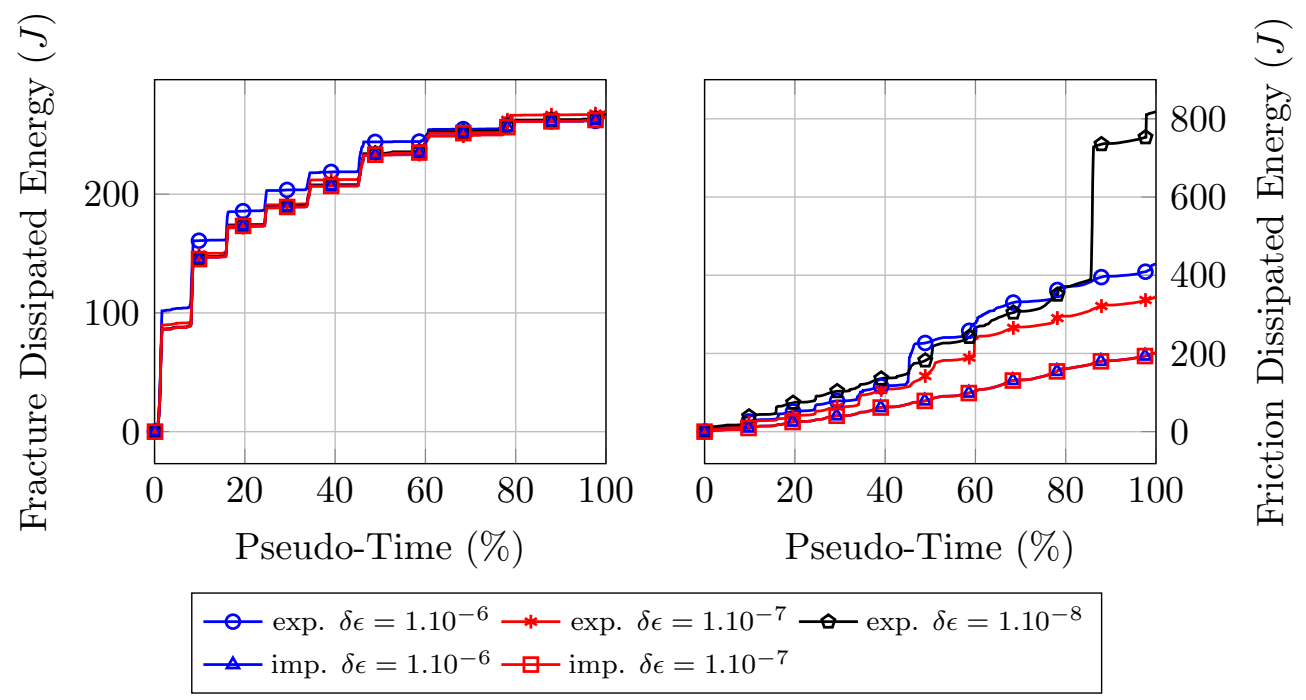

Figure 15: Evolution of the dissipated energy through fracture (left) and friction (right) during the cyclic test

Once more, explicitly computed results, here dissipated energies (fig. 15) by the fracture mechanism, converge toward implicit results. Dissipated energies have similar ranges of values independently of the strain increment. Dissipated energies by the friction mechanism, on the contrary, have a wider range of values. Furthermore an important jump of energy dissipation occurs between $80 \%$ and $90 \%$ of the application of the load for $\delta \epsilon=1.10^{-8}$. At this interval of pseudo-time, the specimen is being unloaded, the global solicitation state switches from tension to compression. Since the specimen is already well damaged, the jump can most likely be explained by an integration error due to the simultaneous initiation of numerous contacts. Nevertheless evolutions of the energy dissipation through friction are similar to the implicitly obtained one, but unlike the fracture dissipated energy, no significant convergence of the values can be found. 
vergence with strain increment refinement of the dissipated energy through friction was not ensured. Here is a first limitation of explicit integration since refining the strain increment does not solve the quantitativeness issue.

On the opposite, implicit integration did not display any difficulties while computing iteratively equilibrium of contact, friction and cohesion forces. The residual error (fig.16b) remains during most of the simulation under the value of $10^{-6}$. Only at transitions from tension to compression state at high level of damage of the sample, the implicit algorithm shows more difficulties to converge, but the residual error remains under the value of $10^{-5}$.

Regarding computation costs (fig.16a), explicit integration is not significantly faster than implicit integration, and computation costs seem to stabilize with finer strain increments.

\subsection{Compression test: results and comments}

Simulations are carried out for strain increments of $\delta \epsilon=7.10^{-5}, \delta \epsilon=$ $7.10^{-6}, \delta \epsilon=7.10^{-7}$ and $\delta \epsilon=7.10^{-8}$. Regarding implicit integration, only simulations for strain increments of $\delta \epsilon=7 \cdot 10^{-5}$ and $\delta \epsilon=7 \cdot 10^{-6}$ are shown since no dependence to the strain increment size is observed.

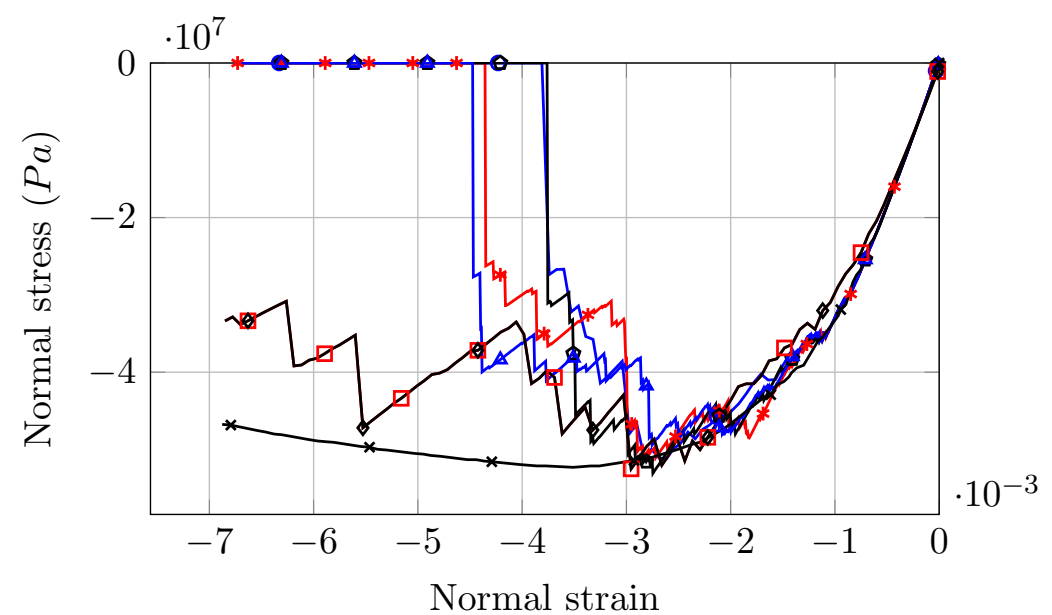

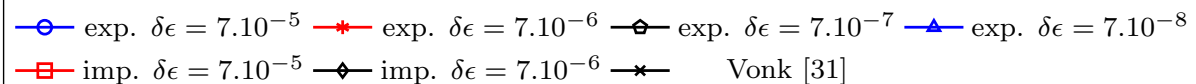

Figure 17: Evolution of the normal stress with respect to normal strain during the whole compression test

The stress-strain response under compression with dry platen, namely 
with high friction at the extremities, is quite ductile (fig.11). Once parameters identified, the LDEM allows to represent correctly this behavior (fig.17). However we can observe that the quality of the computed response is highly dependent on the chosen integration scheme. Whatever the chosen strain increment, explicitly computed responses are clearly too brittle, the complete failure of the specimens occurs soon after the peak-load, while the implicitly computed responses are more ductile. The explicit responses, for different strain increments, are quite close, peak-loads are identical and complete failure always occur in the close range of strains from $3 \cdot 5 \cdot 10^{-3}$ to $4.5 \cdot 10^{-3}$. Although particular convergence issues are not observed, at high strain levels, the explicit solutions remain quite far from the solutions found with implicit integration or experimentally.

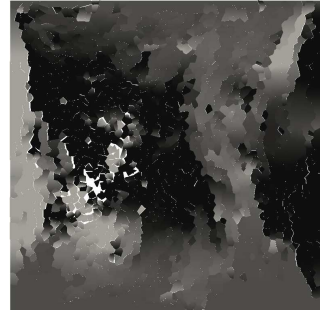

(a) exp.: $\delta \epsilon=7.10^{-5}$

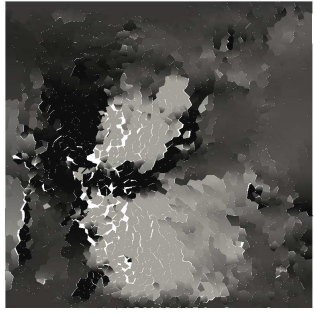

(b) exp.: $\delta \epsilon=7.10^{-6}$

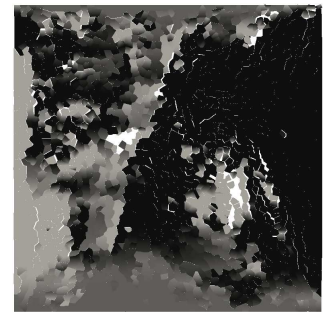

(c) exp.: $\delta \epsilon=7.10^{-7}$

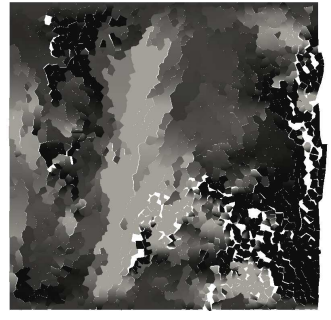

(d) exp.: $\delta \epsilon=7.10^{-8}$

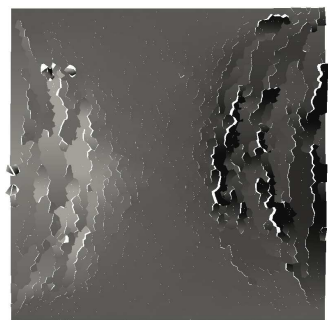

(e) imp.: $\delta \epsilon=7.10^{-5}$

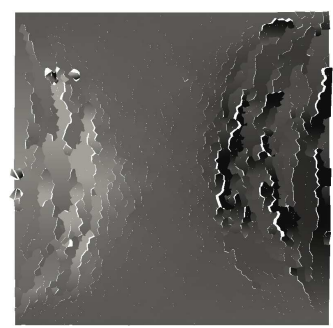

(f) imp.: $\delta \epsilon=7.10^{-6}$

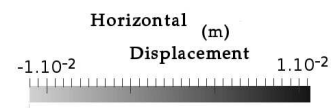

Figure 18: Cracking patterns at failure in compression

Nonetheless, computations of dissipated energy by fracture (fig.19) show higher values for explicit integration than implicit integration. At the end of the simulations, we can see that explicitly computed cracking patterns (fig.18) are irregular and disordered due to the final brittle failure of the specimens, while the implicitly computed ones show classic cracking patterns observed under compressive failure. Explicit specimens appear to have 
collapsed entirely without any specific localization of the cracking, explaining the higher values of dissipated energy by fracture.

In the end, the post-peak behavior of explicitly computed solutions is quite chaotic due to the failure of an important amount of cohesive links. The frictional sliding being possibly activated all over the specimen in absence of cohesive links, computed dissipated energies by friction are also completely erroneous (fig.20).

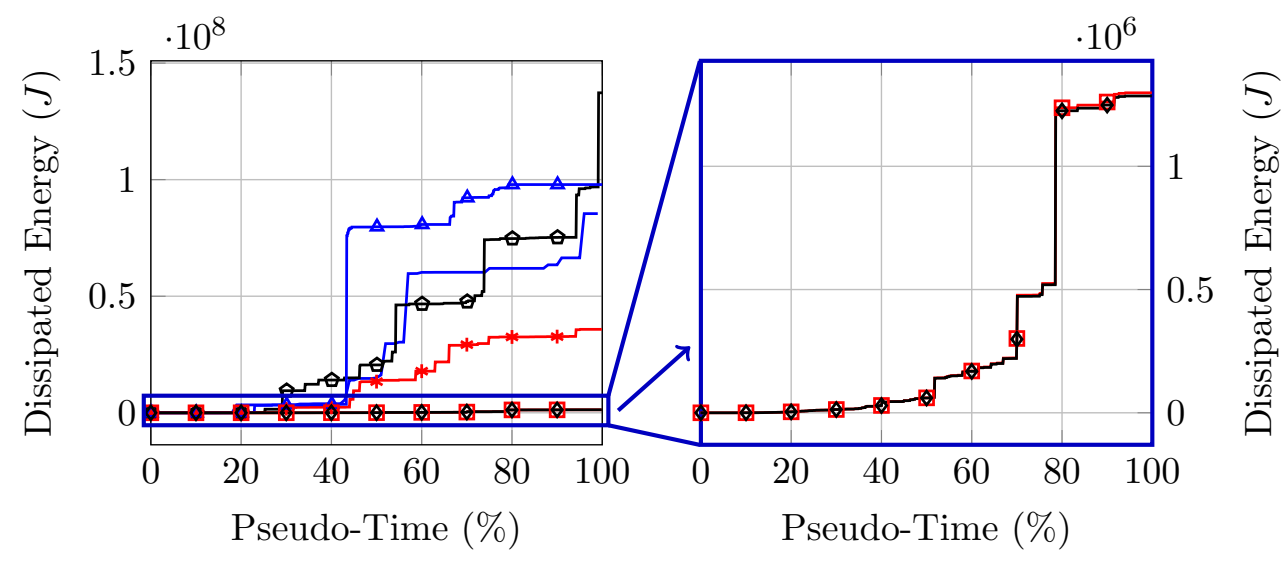

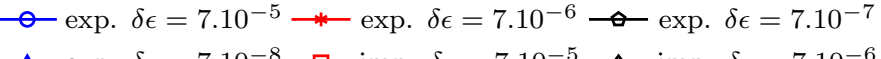
$\leadsto$ exp. $\delta \epsilon=7.10^{-8} \multimap$ imp. $\delta \epsilon=7.10^{-5} \leadsto$ imp. $\delta \epsilon=7.10^{-6}$

Figure 19: Evolution of the dissipated energy by fracture during the whole compression test 


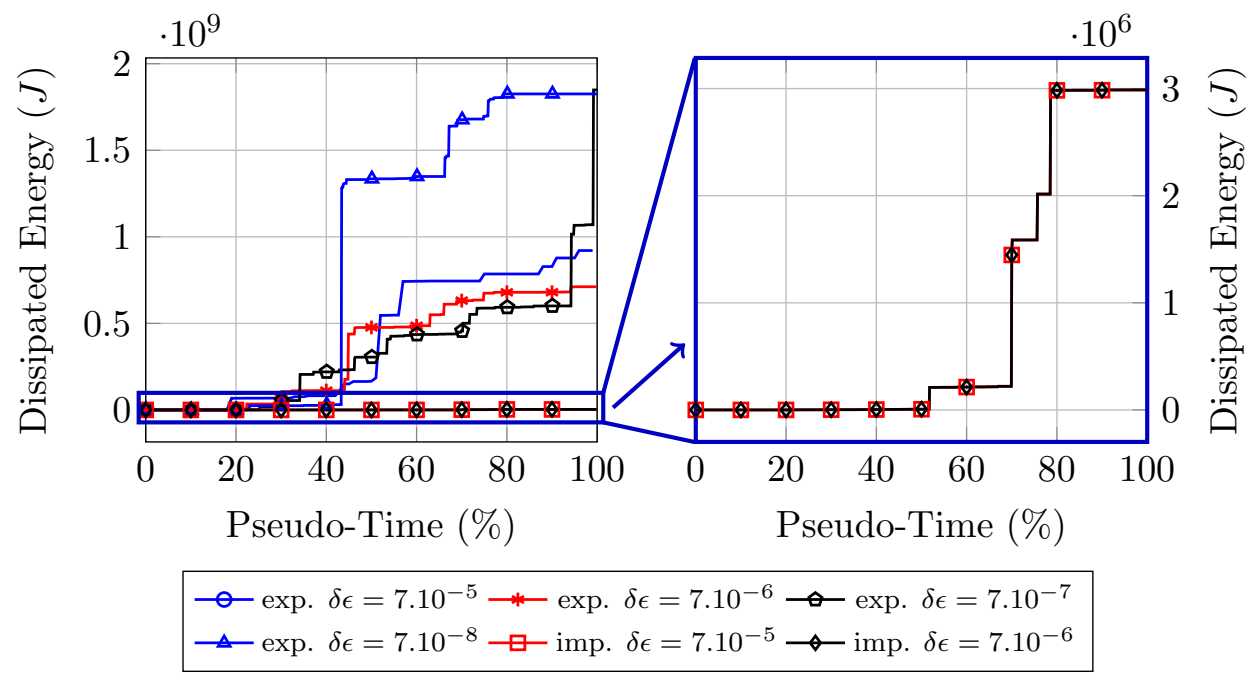

Figure 20: Evolution of the dissipated energy by friction during the whole compression test

At first, during the diffuse cracking part, mainly fracture is active, however after the coalescence of the diffuse microscopic cracks into macroscopic cracks parallel to the applied load, fracture and contact mechanisms are strongly interacting and the propagation of the macroscopic cracks is highly influenced by contact and friction mechanisms of the cracks lips. Therefore if the solution of the contact and friction problems is not accurately computed, direct consequences are observed on the cracking patterns and post-peak behavior of the specimen. All the more as an important number of contact are initiated; and as we observed during the development of the implicit integration scheme, contact forces can be easily miscalculated if thorough attention is not paid to integration. 


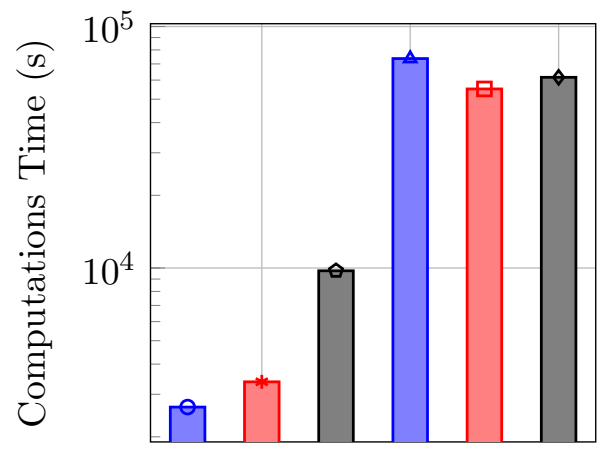

Strain increment and Integration scheme

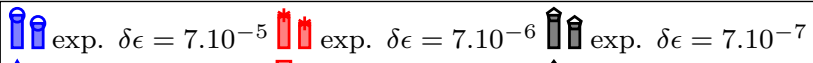

$$
\begin{aligned}
& \text { ปै } \text { exp. } \delta \epsilon=7 \cdot 10^{-8} \text { 目 imp. } \delta \epsilon=7 \cdot 10^{-5} \text { imp. } \delta \epsilon=7 \cdot 10^{-6}
\end{aligned}
$$

(a) Time computation costs

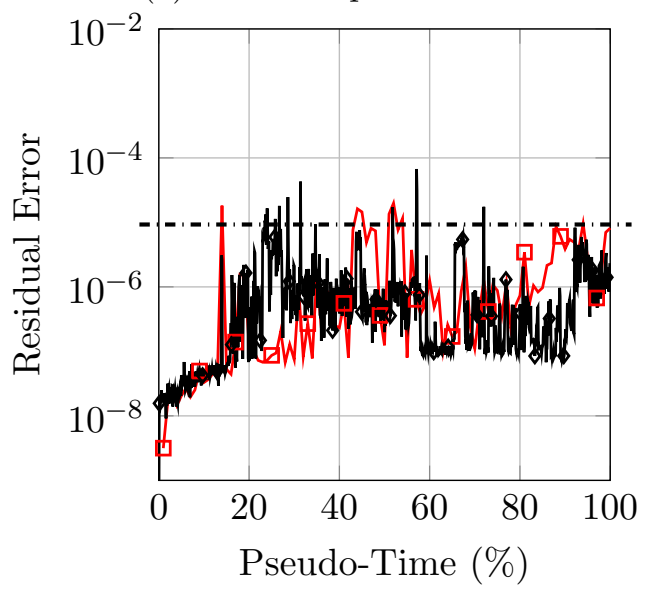

๑一 imp. $\delta \epsilon=7.10^{-5} \multimap$ imp. $\delta \epsilon=7 \cdot 10^{-6}$

(b) Residual error

Figure 21: Time computation costs and residual error of implicit integration of the compression test

In the present case of the compression test, the necessity of implicit integration does not reveal in terms of computation costs (fig.21a). In fact implicit integration is almost as long as explicit integration with a strain increment $\delta \epsilon=1.10^{-8}$.

Nevertheless the use implicit integration for compression tests is inevitable. 
Response of concrete specimens under compression until complete failure could only be followed accurately using implicit integration. The decrease of quantitativeness in the computation of contact forces due to explicit integration, whatever the chosen strain increment, leads to a complete loss of qualitativeness of the computed response, which appears by a non-physical and brittle failure of the specimen. An iterative integration of forces equilibrium seems prerequisite to overcome oscillations issues, generated by nonlinearities at the initiation of contact and amplified by the absence of viscous damping.

Besides, in addition to an unconditional convergence and a better accuracy of the results, the implicit integration does not happen to have robustness issues as the residual error rarely exceeds $10^{-5}$ (fig.21b).

\section{Conclusion}

It is well known that explicit "Saw-tooth" integration is accurate and sufficient when monotonic loading paths and opening cracking modes (mode I or mixed modes I and II) are simulated. However for further use of lattice models for multi-axial, and more specifically sliding cracking modes (e.g. mode II or III in 3D), or cyclic loading paths, the predominance of contact and friction might limit the use of explicit integration.

We addressed in the present paper the question of the validity of explicit integration for such loading paths, which is known to have limited efficiency in the case of strong reversible non-linear behaviors such as contact.

In order to circumvent these issues we proposed an implicit quasi-static integration scheme. The implicit integration scheme is based on fracture and contact mechanisms description. It consists in a combination of the "Saw-tooth" algorithm [20], classically used in explicit integration schemes to manage fracture evolution while ensuring stability; and of a predictorcorrector method, to compute forces equilibrium. A validation of the introduced predictor-corrector method has been realized on a local simulation of a particle subjected to multiple irregular contacts. The additional use of numerical relaxation helped to avoid arising robustness issues.

Since quasi-static framework was considered, disconnected particles from the main structure had to be handled specifically to avoid rigid-body motions. Detection of loose particles is proceeded with a breadth-first search algorithm from graph's theory. The actual treatment consists in removing disconnected 
particles from the structure for robustness purposes. The hypothesis justifying this treatment, that is disconnected particles usually fall away from the structure and do not interact anymore, as for compressive failure, is abusively simplifying. Supplementary efforts would have to be pursued to account for disconnected particles without extensive loss of robustness.

The evaluation of the efficiency of explicit integration has been done on a uniaxial cyclic tension test and a compression test. The simulations with explicit "Saw-tooth" integration of the uniaxial cyclic test showed relatively accurate mechanical response and energy dissipation, while the simulation of the compression test failed from the description of the mechanical behavior. We observed a significant dependency of the computed results to the chosen strain increment, up to non-convergence of the results.

More specifically as far as fracture mechanisms were concerned, independence with load increment refinement and accuracy of the results was obtained, thanks to the "Saw-tooth" algorithm.

However when contact and friction were involved, such convergence was either not reached or reached toward erroneous values, as shown during compression test simulations. The main explanation is the absence of viscous damping due to the quasi-static framework, which provokes oscillations of contacting particles. Therefore when contact and frictions mechanisms are predominant, simulations results can suffer a loss of quantitativeness as well as qualitativeness, implying a loss of physical meaning independently of the time-steps size with explicit integration.

On the contrary the developed implicit integration scheme showed strain increment independence and accuracy of the results even with large increments, while not revealing any particular robustness issues.

Furthermore LDEM computations being time-consuming, implicit integration showed advantages to compute long simulations such as cyclic tests, in which case small enough strain increments to reach convergence and accuracy of the results could hardly be used within reasonable amounts of time.

To conclude, explicit integration has shown its limits in the case of complex loading paths, as soon as contact mechanisms become predominant and even more when contact and fracture mechanisms are simultaneously activated. Moreover, since lattice models can have a vocation for numerical calibration [17] of macroscopic continuous models of quasi-brittle materials [33], quantitativeness of the provided results is essential, in particular scalar 
results such as dissipated energies, which are often used as input parameters of continuous models.

Consequently in such situations, when contact and friction mechanisms are prevailing, implicit integration should be used by means of algorithms such as the integration scheme developed in this paper.

\section{Acknowledgments}

Authors are grateful for the financial support of the French Sustainable Energies and Atomic Energy Commission (CEA) and the ILMAB project (Grant FUI11). The research reported in this paper/article has been supported in part by the SEISM* Paris Saclay Research Institute. 


\section{References}

[1] Cundall, P.A. and Strack, O.D.L. A discrete numerical model for granular assemblies. Géotechnique (1979) 29:47-65.

[2] Kawai, T. New discrete models and their application to seismic response analysis of structures. Nuclear Engineering and Design (1978) 48:207229.

[3] Kikuchi, A., Kawai, T. and Suzuki, N. The rigid bodies-spring models and their applications to three-dimensional crack problems. Computers \& Structures (1992) 44:469-480.

[4] Schlangen, E. and Garboczi, E.J. New method for simulating fracture using an elastically uniform random geometry lattice. International Journal of Engineering Science (1996) 34:1131-1144.

[5] Bolander, J.E. and Saito, S. Fracture analyses using spring networks with random geometry. Engineering Fracture Mechanics (1998) 61:569591.

[6] van Mier, J.G.M., van Vliet, M.R.A. and Wang, T.K. Fracture mechanisms in particle composites: statistical aspects in lattice type analysis. Mechanics of Materials (2002) 34:705-724.

[7] Herrmann, H.J. and Roux, S. (eds.) Statistical Models for the Fracture of Disordered Media. North Holland, Amsterdam, The Netherlands (1990).

[8] Shi, G. and Goodman, R.E. Generalization of two-dimensional discontinuous deformation analysis for forward modeling. International Journal for Numerical and Analytical Methods in Geomechanics (1989) 13:359-380.

[9] Hentz, S., Daudeville, L. and Donzé, F.V. Identification and Validation of a Discrete Element Model for Concrete. Journal of Engineering Mechanics (2004) 130:709-719.

[10] Tillemans, H.J. and Herrmann, H.J. Simulating deformations of granular solids under shear. Physica A: Statistical Mechanics and its Applications (1995) 217:261-288. 
[11] D'Addetta, G.A., Kun, F. and Ramm, E. On the application of a discrete model to the fracture process of cohesive granular materials. Granular Matter (2002) 4:77-90.

[12] Issa, J.A. and Nelson, R.B. Numerical Analysis of Micromechanical Behavior of Granular Materials. Engineering Computations (1992) 9:211223.

[13] Kun, F. and Herrmann, H.J. A study of fragmentation processes using a discrete element method. Computer Methods in Applied Mechanics and Engineering (1996) 138:3-18.

[14] Moreau, J.J. Unilateral contact and dry friction in finite freedom dynamics. CISM Courses and Lectures (1988) Nonsmooth Mechanics and Applications:1-82.

[15] Shi, G.H. and Goodman, R.E. Two dimensional discontinuous deformation analysis. International Journal for Numerical and Analytical Methods in Geomechanics (1985) 9:541-556.

[16] Jing, L. Formulation of discontinuous deformation analysis (DDA)an implicit discrete element model for block systems. Engineering Geology (1998) 49:371-381.

[17] Delaplace, A. and Desmorat, R. Discrete 3D model as complimentary numerical testing for anisotropic damage. International Journal of Fracture (2008) 148:115-128.

[18] Moukarzel, C. and Herrmann, H.J. A vectorizable random lattice. Journal of Statistical Physics (1992) 68:911-923.

[19] Oliver, J., Huespe, A.E. and Cante, J.C. An implicit/explicit integration scheme to increase computability of non-linear material and contact/friction problems. Computer Methods in Applied Mechanics and Engineering (2008) 197:1865-1889.

[20] Rots, J.G. and Invernizzi, S. Regularized sequentially linear saw-tooth softening model. International Journal for Numerical and Analytical Methods in Geomechanics (2004) 28:821-856. 
[21] Oliver-Leblond, C., Delaplace, A., Ragueneau, F. and Richard, B. Nonintrusive global/local analysis for the study of fine cracking. International Journal for Numerical and Analytical Methods in Geomechanics (2013) 37:973-992.

[22] Perkins, E. and Williams, J.R. A fast contact detection algorithm insensetive to object size. Engineering Computations (2001) 18:48-61.

[23] O'Rourke, J., Chien C.B., Olson, T. and Naddor, D. A new linear algorithm for intersecting convex polygons. Computer Graphics and Image Processing (1982) 19:384-391.

[24] Feng, Y.T. and Owen, D.R.J. A 2D polygon/polygon contact model: algorithmic aspects. Engineering Computations (2003) 21:265-277.

[25] Cusatis, G., Mencarelli, A., Pelessone, D. and Baylot, J. Lattice Discrete Particle Model (LDPM) for failure behavior of concrete. II: Calibration and validation. Cement and Concrete composites (2011) 33:891-905.

[26] Rots, J.G., Belletti, B. and Invernizzi, S. Robust modeling of RC structures with an "event-by-event" strategy. Engineering Fracture Mechanics (2008) 75:590-614.

[27] Simo, J. C. and Laursen, T. A. An augmented Lagrangian treatment of contact problems involving friction. Computers and Structures (1992) 42:97-116.

[28] Moore, E.F. The shortest path through a maze. In Proceedings of the International Symposium on the Theory of Switching, Harvard University Press (1959) 292-285.

[29] Rossi, P., Ulm, F.-J. and Hachi, F. Compressive Behavior of Concrete: Physical Mechanisms and Modeling. Journal of Engineering Mechanics (1996) 122:1038-1043.

[30] Cornelissen, H. A. W., Hordijk, D. A. and Reinhardt, H. W. Experimental determination of crack-softening characteristics of normalweight and lightweight concrete. Heron (1986) 31:45-56.

[31] Vonk, R.A. Softening of concrete loaded in compression. Eindhoven University of Technology, The Netherlands (1992). 
[32] Rinaldi, A. and Lai, Y.C. Statistical damage theory of 2D lattices: energetics and physical foundations of damage parameter. International Journal of Plasticity (2007) 23:1796-1825.

[33] Richard, B. and Ragueneau, F. Continuum damage mechanics based model for quasi brittle materials subjected to cyclic loadings: Formulation, numerical implementation and applications Engineering Fracture Mechanics (2013) 98:383-406. 